\title{
RANKING DE ARTÍCULOS MÁS LEÍDOS DE LA URACCAN PUBLICADOS EN SUS REVISTAS ELECTRÓNICAS CON ACCESO ABIERTO
}

Juan Francisco Perera Lumbí ${ }^{[1]}$

\section{Resumen}

Este estudio muestra el ranking de los artículos más leídos de la Revista Ciencia e Interculturalidad y la Revista Universitaria Caribe; así ha inducir una reflexión interna en las universidades nicaragüenses, con énfasis en la Universidad de las Regiones Autónomas de la Costa Caribe Nicaragüense (URACCAN), sobre las ventajas de la difusión de resultados de investigaciones desde las revistas electrónicas tanto en el uso de acceso abierto y de software de código abierto como Open Journal Systems (OJS).

Entre los resultados sobresalen: i) La revista digital Ciencia e Interculturalidad Incorporada en el Portal de Revistas Nacionales de Nicaragua, Portal del Sistema Regional de Información en Línea para revistas científicas de América Latina, el Caribe y Portugal (LATINDEX) y en el portal de Latin American Journals Online (LAMJOL). ii) Ciencia e Interculturalidad, en Latin American Jornal Online (LAMJOL) recibió un total de 53,757 visitas desde marzo 2011 a octubre 2013; en el Portal de Revistas de Nicaragua recibió un total de 12,522 visitas en revisión a resúmenes y 13,881 para revisar o descargar artículo completo en PDF, del 2012 a noviembre del 2013; y en el Portal de Revistas de URACCAN recibió un total de 18,661 visitas para revisar resúmenes y 32,254 para revisar o descargar artículo completo en PDF, durante junio 2011 a octubre 2013. iii). El Consejo Nicaragüense de Ciencia y Tecnología de Nicaragua (CONICYT), entregó en noviembre del 2013, un reconocimiento a la revista "por su esfuerzo en elevar la calidad y aplicación de los criterios de LATINDEX y lograr visibilidad de la producción científica de Nicaragua en LAMJOL".

Palabras clave: Revistas electrónicas; Ranking, artículos más leídos, revista Ciencia e Interculturalidad; revista Caribe, URACCAN; Nicaragua.

[1] Master en Ciencias Forestales, Director de Investigación y Posgrado de la Universidad de las Regiones Autónomas de la Costa Caribe Nicaragüense (URACCAN). Email: frankperera@yahoo.com 


\section{Summary}

This study shows the ranking of the most read articles in the Science and Interculturality Magazine and the Caribbean University Magazine, which induce and brought across an internal reflection within Nicaraguan universities, with emphasis on the University of the Autonomous Regions of the Nicaraguan Caribbean Coast (URACCAN), regarding the benefits of disseminating research results from electronic magazines by using both open access and open source software code such as Open Journal Systems (OJS).

Among the results we highlight: i) The digital magazine Science and Interculturality, incorporated in the Nicaragua's National Magazine Portal, Portal Online Regional Information System for scientific magazines of Latin America, the Caribbean and Portugal (Latindex) and the portal Latin American Journals Online (Lamjol). ii) Science and Interculturality in Latin American Journals Online (Lamjol) received a total of 53.757 visitors since March 2011 to October 2013; the Portal of Journals of Nicaragua received a total of 12,522 visits that reviewed abstracts and 13,881 to review or download full article in PDF, from 2012 to November 2013. And the Portal of Journals of URACCAN received a total of 18,661 visits to review abstracts and 32,254 to review or to download full article in PDF during June 2011 to October 2013. iii). The Nicaraguan Council of Science and Technology (CONICYT), delivered in November 2013, a recognition to the magazine "for their efforts in raising the quality and application of the LATINDEX criteria's and to achieve visibility of scientific production in LAMJOL - Nicaragua".

Keywords: Electronic journals, ranking, most read articles, magazine of Science and Interculturality; Caribbean magazine, URACCAN; Nicaragua.

\section{Introducción}

Los primeros esfuerzos de la URACCAN para efectuar publicación de los resultados de las investigación en formato de artículos inició en el año de 1999 con la creación de la Revista universitaria Caribe en versión impresa; posteriormente, en el año 2008 se crea la Revista Ciencia e Interculturalidad en formato impreso, de la cual a la fecha hemos publicado 12 números. A inicios del 2011 se crea y publica la versión digital de ambas revistas.

La incursión de la universidad en la publicación científica en formato digital, está estrechamente vinculada al apoyo brindado por el Programa para el Fortalecimiento de la Información para la Investigación (PERI), el cual es un proyecto creado y desarrollado por el Consejo Nacional de Universidades (CNU) que surge en el 2006 como una iniciativa para acompañar y fortalecer el acceso a la información para la investigación dentro del sistema de investigación universitaria nicaragüense. Entre otras cosas, PERI aseguró el proceso de sensibilización y capacitación de directores, 
editores e informáticos vinculados a las revistas, en los procesos de Gestión de Revistas Electrónicas con Open Journal System (OJS).

En marzo del año 2011 se publica el Portal de Revistas de la URACCAN, el que aglutina tres revistas: Ciencia e Interculturalidad, Revista Universitaria Caribe y URACCAN al Día. En abril del 2011, la Revista Ciencia e Interculturalidad ingresa al portal de Latin America Journals Online, sitio web que apoya la publicación en línea de revistas latinoamericanas.

En el mes de marzo del 2011, la versión impresa de Ciencia e Interculturalidad ingresa al catálogo del portal del Sistema Regional de Información en Línea para Revistas Científicas de América Latina, el Caribe y Portugal (LATINDEX), y en mayo del 2012 ingresa la versión digital.

LATINDEX es un sistema de Información sobre las revistas de investigación científica, técnico-profesionales y de divulgación científica y cultural que surgió en 1995 en la Universidad Nacional Autónoma de México (UNAM) y se convirtió en una red de cooperación regional a partir de 1997. Actualmente, LATINDEX ofrece tres bases de datos: 1) Directorio, con datos bibliográficos y de contacto de todas las revistas registradas, ya sea que se publiquen en soporte impreso o electrónico; 2) Catálogo, que incluye únicamente las revistas impresas y electrónicas que cumplen los criterios de calidad editorial diseñados por LATINDEX; 3 ) Enlace a Revistas Electrónicas, que permite el acceso a los textos completos en los sitios en que se encuentran disponibles.

En el año 2012 la Revista Ciencia e interculturalidad ingresa al Portal de Revistas Nacionales de Nicaragua, este portal aglutina solamente las revistas científicas que cumplen los criterios de calidad estipulados por LATINDEX para ingresar al catálogo.

El 12 de noviembre del 2013, el Consejo Nicaragüense de Ciencia y Tecnología de Nicaragua (CONICYT), entregó a la Universidad de las Regiones Autónomas de la Costa Caribe Nicaragüense (URACCAN), un reconocimiento a la revista científica Ciencia e Interculturalidad, "por su esfuerzo en elevar la calidad y aplicación de los criterios del Sistema Regional de Información en Línea, para revistas científicas de América Latina, el Caribe, España y Portugal (LATINDEX) y lograr visibilidad de la producción científica de Nicaragua en Latin American Journal OnLine (LAMJOL)". Las otras revistas con reconocimiento del CONICYT fueron: La Calera de la Universidad Nacional Agraria (UNA), NEXO de la Universidad Nacional de Ingeniería (UNI), Encuentro de la Universidad Centroamericana (UCA) y Cultura de paz de la Universidad Politécnica (UPOLI). 


\section{El Portal de Revistas de la URACCAN}

Como se puede apreciar en el cuadro No. 1, el Portal de Revistas de la URACCAN (figura 1) ha sido un éxito completo en materia de difusión de la publicación científica de la Universidad y sus investigadores colaboradores. El monitoreo del período que comprende de junio del 2011 a octubre del 2013 refleja, 38,855 revisiones de resúmenes de artículos y 64,182 revisiones o descargar de artículos completos, lo anterior implica un promedio de 2,292 ingresos mensuales para revisar o descargar los artículos completos y 1,387 para revisar resúmenes.

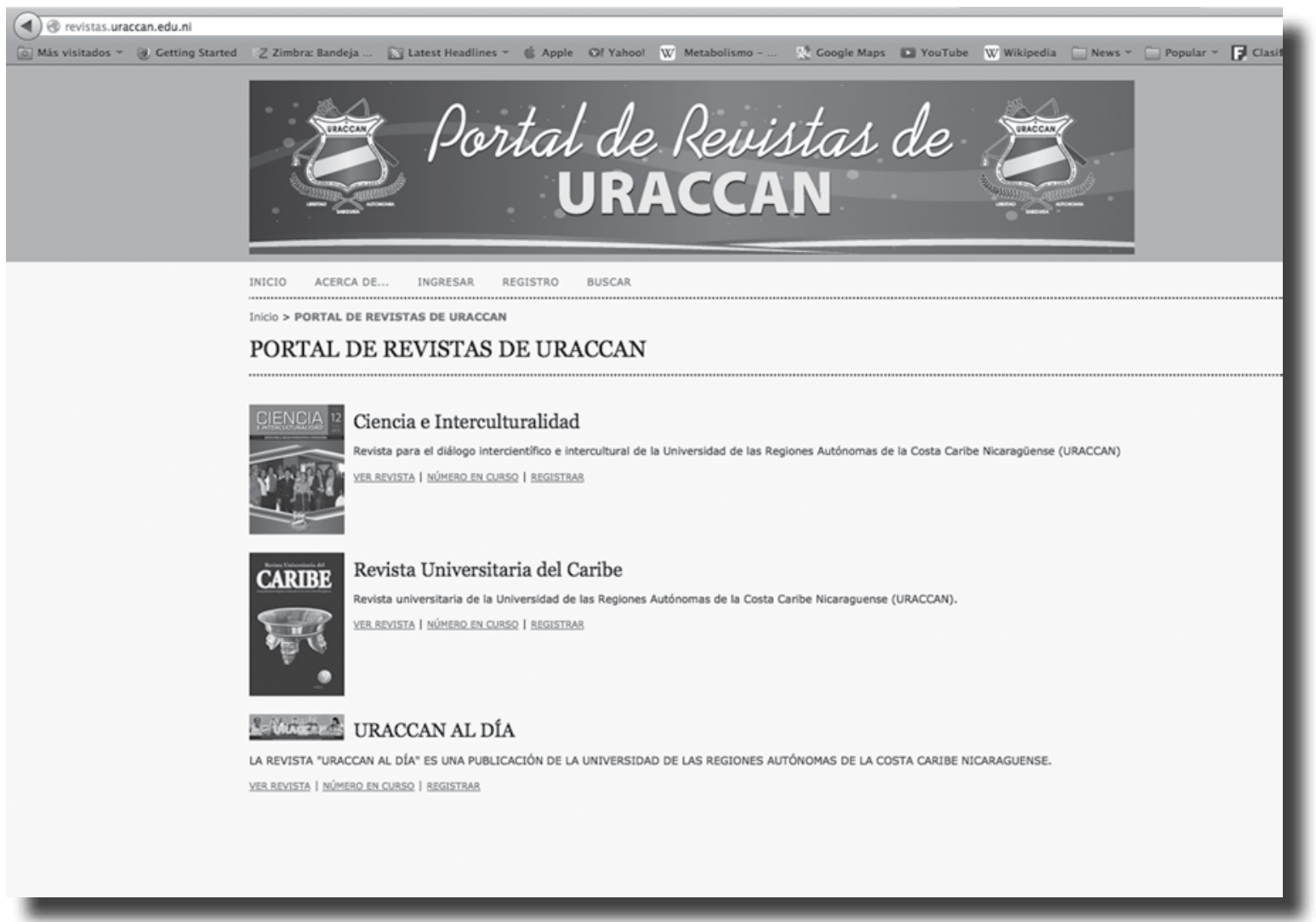

Figura No. 1. Portal de revistas de la URACCAN, disponible en: http://revistas.uraccan.edu.ni/index.php/index 
Cuadro No. 1. Resumen de visitas al Portal de Revistas de la URACCAN período junio 2011 a octubre del 2013

\begin{tabular}{|l|r|r|r|r|r|r|}
\hline \multirow{2}{*}{ Revista } & \multicolumn{7}{|c|}{ Número y tipo de consultas } \\
\cline { 2 - 7 } & Resumen & \multicolumn{1}{c|}{$\%$} & Art completo & \multicolumn{1}{c|}{$\%$} & \multicolumn{1}{c|}{ Total } & \multicolumn{1}{c|}{$\%$} \\
\hline $\begin{array}{l}\text { Ciencia e } \\
\text { Interculturalidad }\end{array}$ & 18,661 & 32 & 32,254 & 50 & 50,915 & 49 \\
\hline Caribe & 12,315 & 32 & 20,819 & 32 & 33,134 & 32 \\
\hline URACCAN al Día & 7,879 & 20 & 11,109 & 17 & 18,988 & 18 \\
\hline Total & 38,855 & 100 & 64,182 & 100 & 103,037 & 100 \\
\hline
\end{tabular}

Si analizamos el comportamiento para cada una de las revistas, Ciencia e Interculturalidad tiene un promedio mensual de 1,152 visitas para revisar o descargar artículos completos y 666 para revisar resúmenes; por su parte, la Revista Caribe tiene un promedio mensual de 1735 visitas para revisar o descargar artículos completos y 440 para revisar resúmenes; la revista URACCAN al Día tiene un promedio mensual de 396 visitas para revisar o descargar artículos completos y 285 para revisar resúmenes.

\section{Ranking de los artículos más consultados de la Revista Ciencia e Interculturalidad en el Portal de Revistas de la URACCAN}

En el cuadro No. 2 se presenta el ranking de los 50 artículos más consultados de la Revista Ciencia e Interculturalidad en el Portal de Revistas de la URACCAN. Evidentemente, los artículos contenidos en números más nuevos tienen desventaja para aparecer entre los más consultados, por el tiempo de permanencia en el sitio.

\section{Ranking de los artículos más consultados de la Revista Caribe en el Portal de Revistas de la URACCAN}

En el cuadro No. 3 se presenta el ranking de los 50 artículos más consultados de la Revista Caribe en el Portal de Revistas de la URACCAN. Evidentemente, los artículos contenidos en números más antiguos tienen desventaja para aparecer entre los más consultados, ya que la alimentación del sitio correspondiente a los números del 1 al 9 se hizo en orden inverso. 
Cuadro No. 2. Ranking de 50 artículos más consultados de la Revista Ciencia e Interculturalidad en el Portal de Revistas de la URACCAN período junio 2011 a octubre del 2013

\begin{tabular}{|c|c|c|c|c|c|c|c|}
\hline $\begin{array}{l}\bar{\Xi} \\
\stackrel{0}{\circ}\end{array}$ & 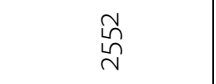 & $\begin{array}{l}\stackrel{2}{\hat{\sigma}} \\
\sim\end{array}$ & 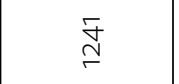 & $\begin{array}{l}\underset{N}{N} \\
\stackrel{0}{ }\end{array}$ & $\begin{array}{l}\infty \\
\stackrel{0}{ } \\
\stackrel{0}{\leftarrow}\end{array}$ & $\begin{array}{l}\hat{b} \\
\stackrel{\circ}{\circ}\end{array}$ & $\begin{array}{l}\infty \\
\infty \\
\infty\end{array}$ \\
\hline 岁 & 离 & 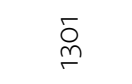 & \begin{tabular}{l}
\multirow{\infty}{*}{} \\
$\infty$
\end{tabular} & $\stackrel{\infty}{\wedge}$ & $\underset{\infty}{\mathbb{\infty}}$ & ก้ & $\stackrel{\circ}{f}$ \\
\hline 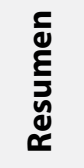 & $\stackrel{\llcorner}{m}$ & $\stackrel{+}{m}$ & $\hat{n}$ & $\stackrel{m}{\tilde{r}}$ & $\stackrel{\bullet}{\circ}$ & $\begin{array}{l}\stackrel{2}{m} \\
m\end{array}$ & $\stackrel{\infty}{\check{\nabla}}$ \\
\hline 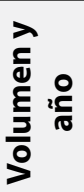 & 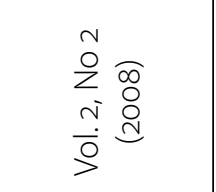 & 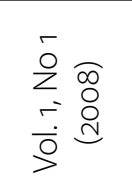 & 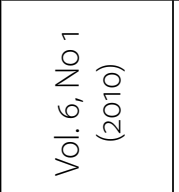 & 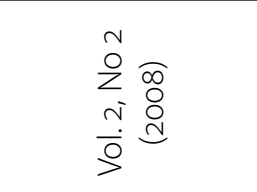 & 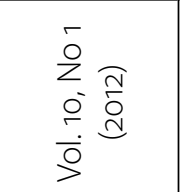 & 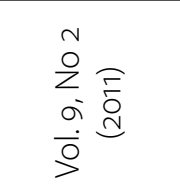 & 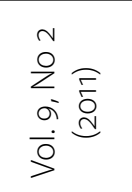 \\
\hline 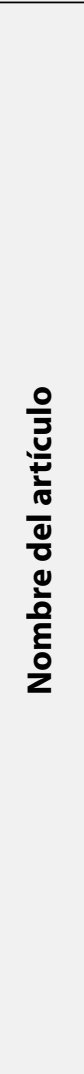 & 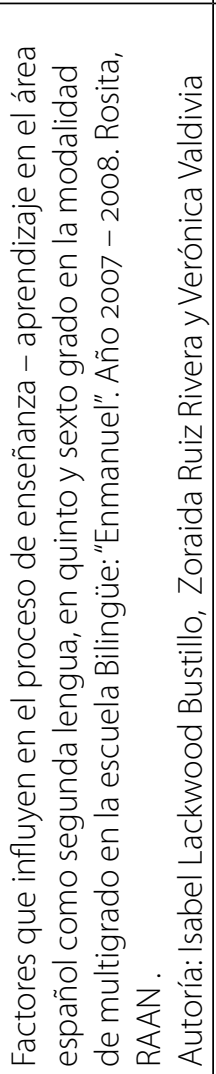 & 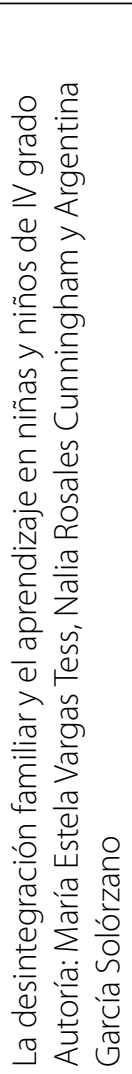 & 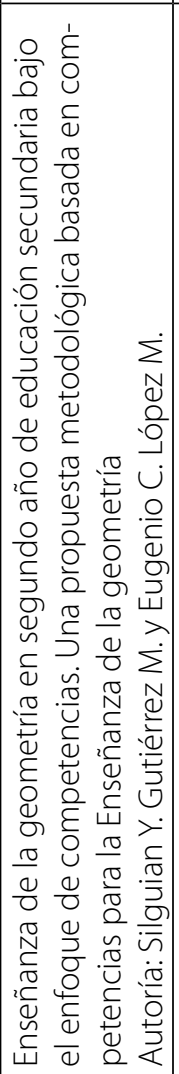 & 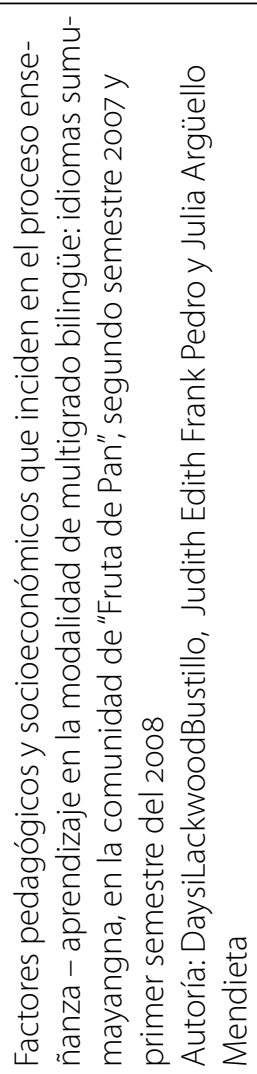 & 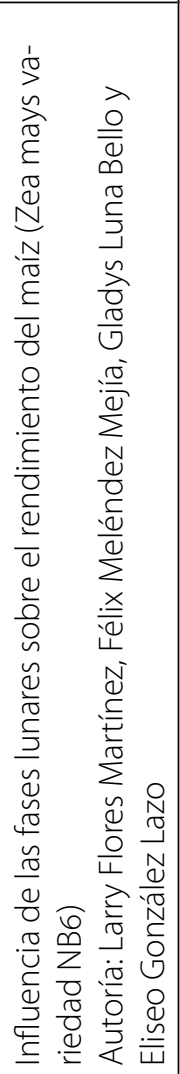 & 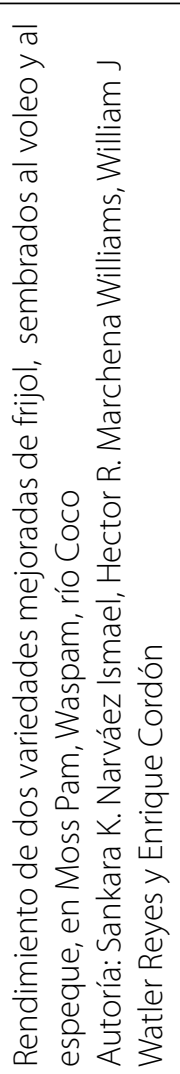 & 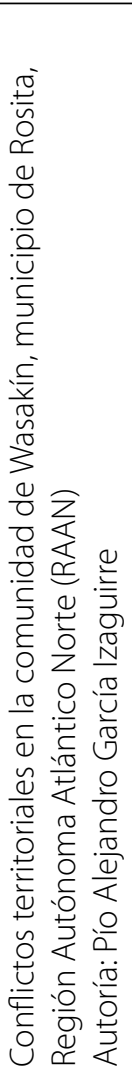 \\
\hline 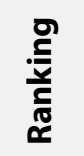 & $\tau$ & $N$ & $m$ & $\nabla$ & $\llcorner n$ & 6 & $\wedge$ \\
\hline
\end{tabular}




\begin{tabular}{|c|c|c|c|c|c|c|c|c|c|}
\hline $\begin{array}{l}\bar{\Xi} \\
\stackrel{0}{\circ}\end{array}$ & $\stackrel{\hat{n}}{\infty}$ & ఫે & $\stackrel{n}{N}$ & $\stackrel{\llcorner n}{\nwarrow}$ & ஜ & $\stackrel{\text { L }}{\wedge}$ & ชู & $\begin{array}{l}0 \\
\infty \\
0\end{array}$ & $\overline{6}$ \\
\hline 㟔 & ⿵人一 & $\stackrel{\substack{m \\
m}}{n}$ & 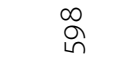 & $\stackrel{\substack{m \\
m}}{2}$ & $\begin{array}{l}+ \\
\text { o }\end{array}$ & $\stackrel{\infty}{\underset{m}{m}}$ & 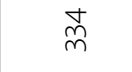 & $\stackrel{b}{\stackrel{n}{*}}$ & $\stackrel{\ln }{f}$ \\
\hline 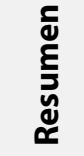 & $\stackrel{b}{\sim}$ & d্ & 守 & $\stackrel{m}{m}$ & 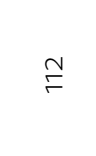 & $\hat{n}$ & $\stackrel{\substack{n \\
m}}{m}$ & $\stackrel{m}{\sim}$ & $\stackrel{b}{\bar{v}}$ \\
\hline 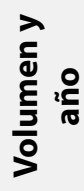 & 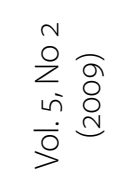 & 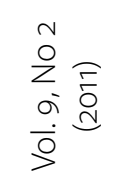 & 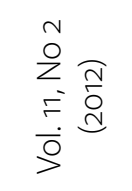 & 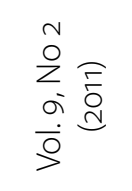 & 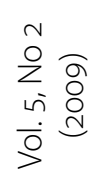 & 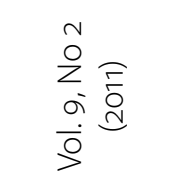 & 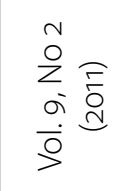 & 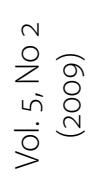 & 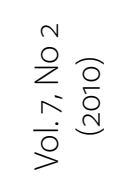 \\
\hline 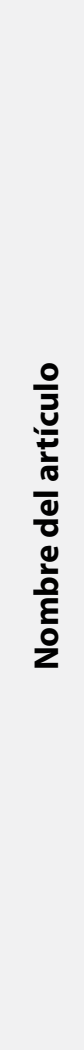 & 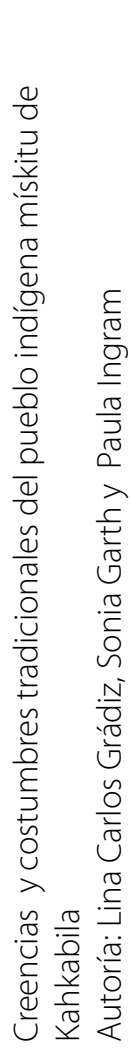 & 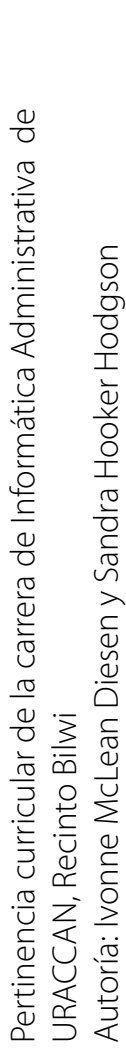 & 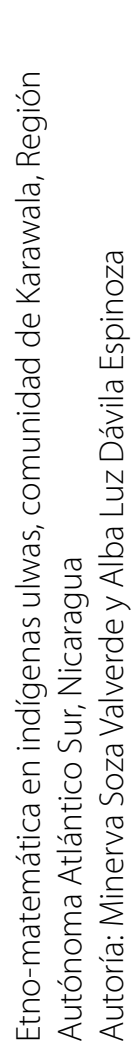 & 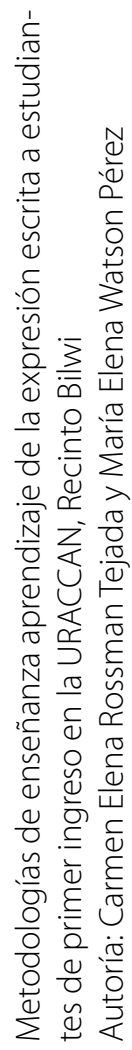 & 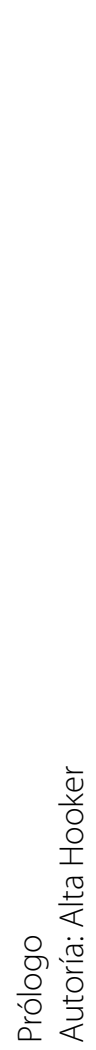 & 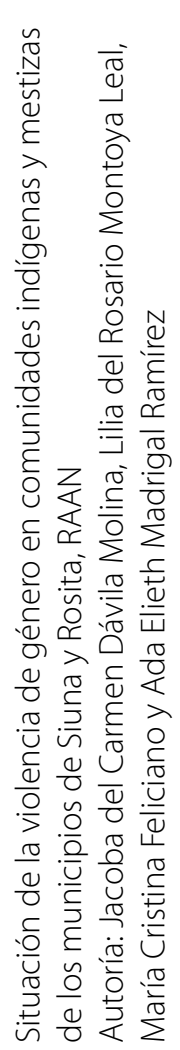 & 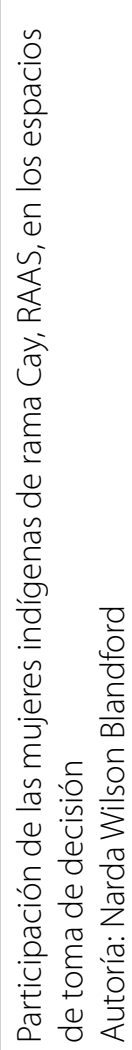 & 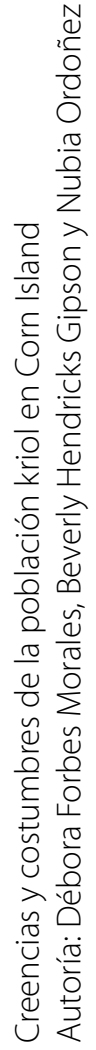 & 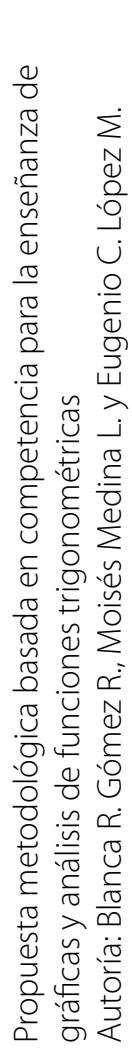 \\
\hline$\frac{.5}{\frac{5}{c}}$ & $\infty$ & $a$ & $\stackrel{\circ}{\circ}$ & $F$ & $\stackrel{\mathcal{N}}{ }$ & $\stackrel{m}{r}$ & $\underset{\leftarrow}{ }$ & $\stackrel{\sim}{\sim}$ & $\bullet$ \\
\hline
\end{tabular}




\begin{tabular}{|c|c|c|c|c|c|c|c|c|c|}
\hline $\begin{array}{l}\overline{\mathbb{8}} \\
\stackrel{0}{\circ}\end{array}$ & $\hat{n}$ & ดे & ర্ & బิ & $\hat{\widehat{\sigma}}$ & ชి & $\begin{array}{l}\curvearrowright \\
\infty \\
\mapsto n\end{array}$ & 㐫 & 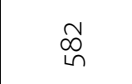 \\
\hline 㟔 & $\frac{d}{d}$ & $\stackrel{\sim}{m}$ & 导 & 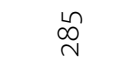 & $\stackrel{+}{\underset{\sim}{*}}$ & ֶै & $\stackrel{\ominus}{\stackrel{+}{+}}$ & ô & $\stackrel{m}{n}$ \\
\hline 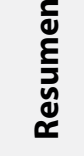 & $\stackrel{\curvearrowright}{\sim}$ & 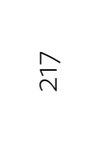 & 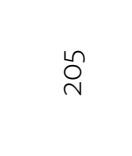 & $\stackrel{\text { qे }}{m}$ & 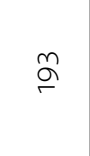 & $\stackrel{\text { fo }}{\ulcorner}$ & $\stackrel{\stackrel{M}{E}}{\sim}$ & $\underset{\sim}{\stackrel{1}{ }}$ & ڤે \\
\hline 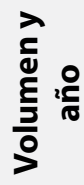 & 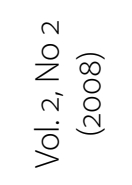 & $\begin{array}{l}\bar{o} \\
\frac{2}{z} \\
\infty \\
\infty \\
\overline{0} \\
\overline{0}\end{array}$ & 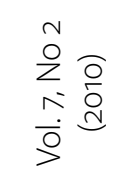 & 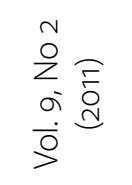 & 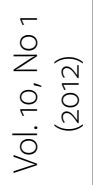 & 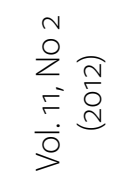 & 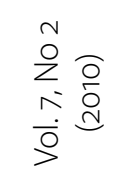 & 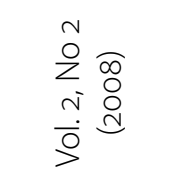 & 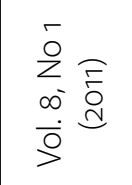 \\
\hline 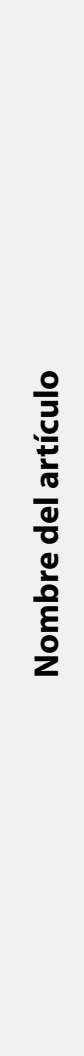 & 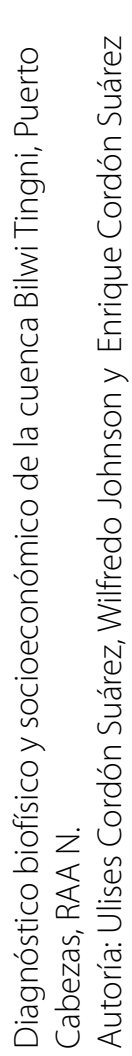 & 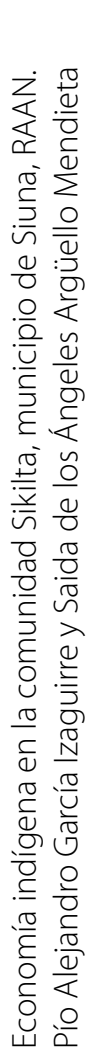 & 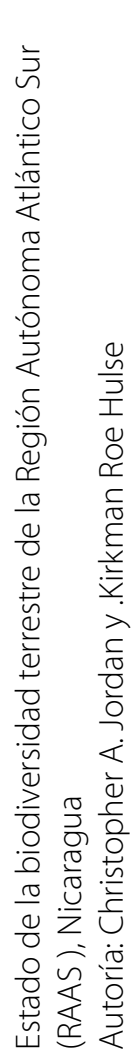 & 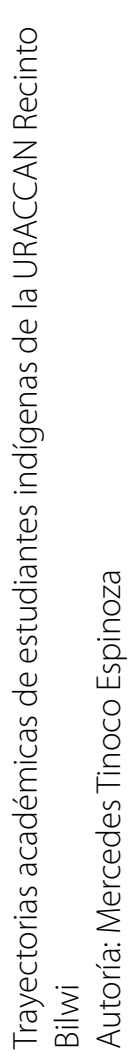 & 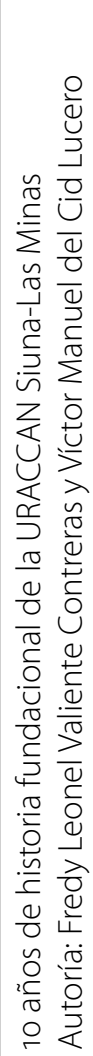 & 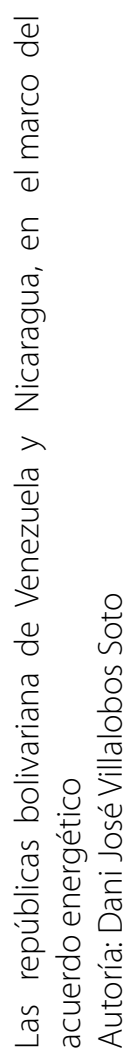 & 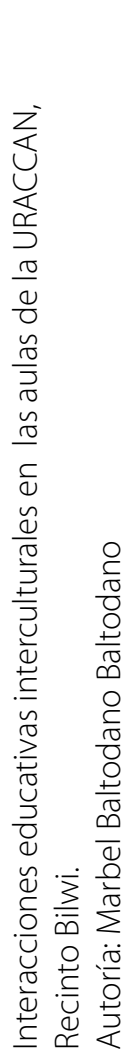 & 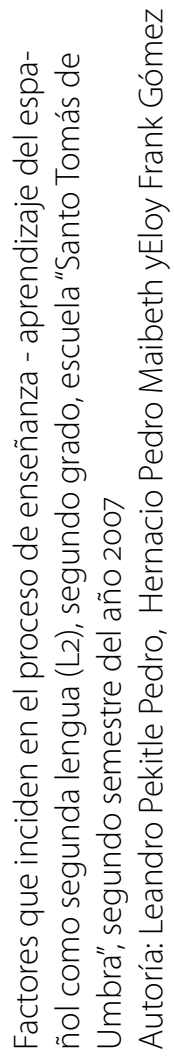 & 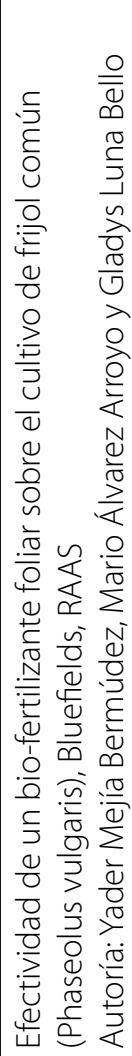 \\
\hline 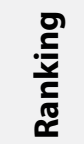 & $\stackrel{F}{ }$ & $\stackrel{\infty}{\sim}$ & $\stackrel{\rho}{\rho}$ & ป & $\bar{v}$ & ㄱ & $\stackrel{\sim}{\sim}$ & ป & $\stackrel{\llcorner}{\sim}$ \\
\hline
\end{tabular}




\begin{tabular}{|c|c|c|c|c|c|c|c|c|c|}
\hline $\begin{array}{l}\bar{\Xi} \\
\stackrel{0}{\circ}\end{array}$ & $\stackrel{b}{n}$ & $\stackrel{b}{n}$ & 周 & ర్ & $\stackrel{\text { }}{\sigma}$ & 吕 & $\stackrel{\bar{\infty}}{\forall}$ & $\begin{array}{l}\stackrel{0}{\infty} \\
+\end{array}$ & $\hat{n}_{\sigma}^{\hat{\sigma}}$ \\
\hline 㟔 & $\stackrel{\circ}{+}$ & $\stackrel{\frac{N}{m}}{2}$ & ָ̃ & $\stackrel{\bar{\alpha}}{\sim}$ & $\frac{b}{m}$ & $\stackrel{\sim}{m}$ & ๙ิ & ô & 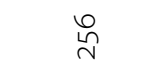 \\
\hline 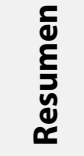 & $\begin{array}{l}\text { ㅇ } \\
\stackrel{\sigma}{-}\end{array}$ & $\underset{\sim}{\stackrel{ন}{N}}$ & ঃ & $\stackrel{\text { సે }}{\text {. }}$ & $\stackrel{a}{2}$ & $\ddot{n}$ & $\underset{\ulcorner}{+}$ & $\underset{\sim}{\stackrel{\sim}{r}}$ & 음 \\
\hline 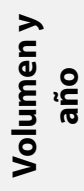 & 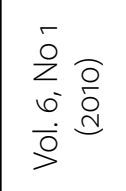 & 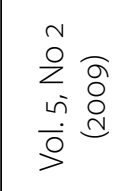 & 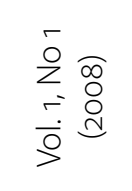 & 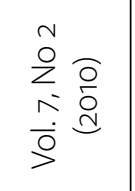 & 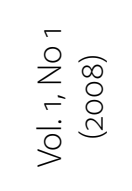 & 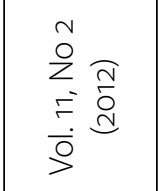 & 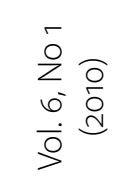 & 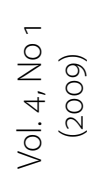 & 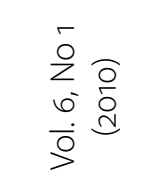 \\
\hline 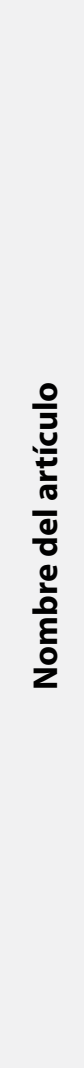 & 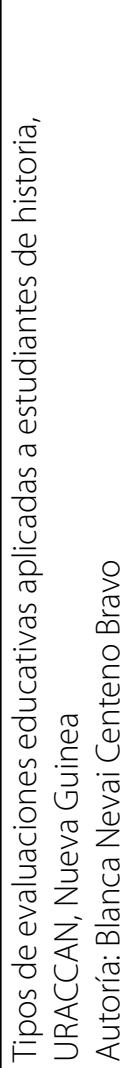 & 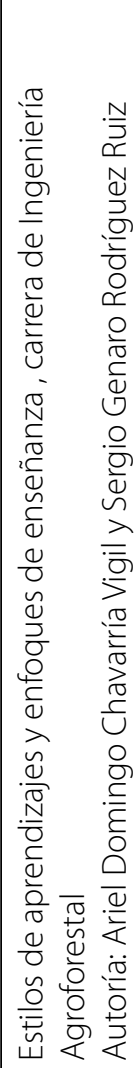 & 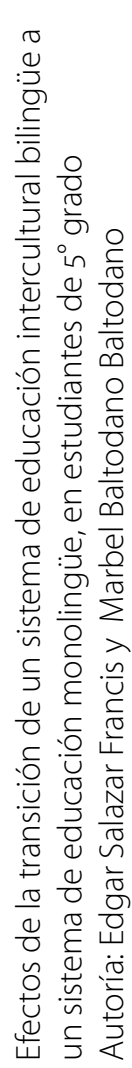 & 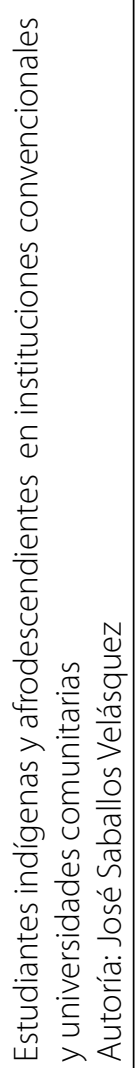 & 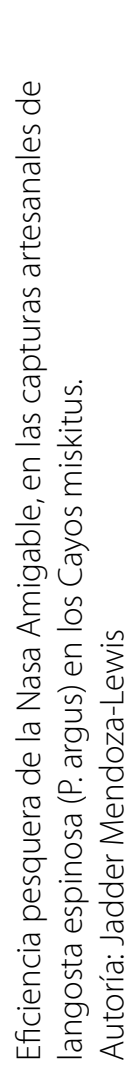 & 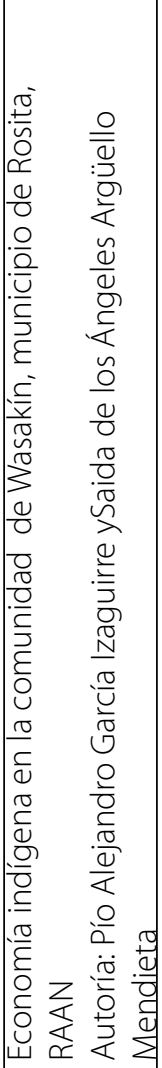 & 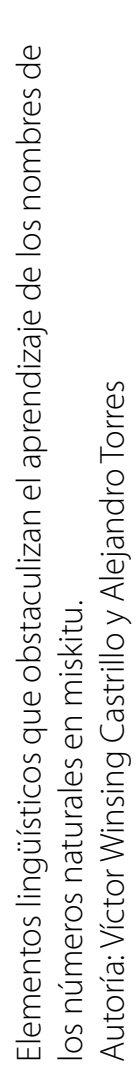 & 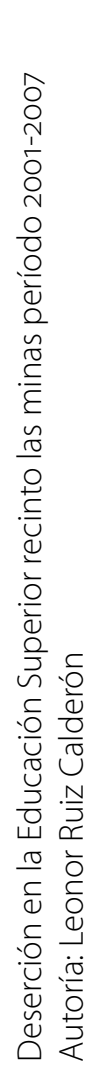 & 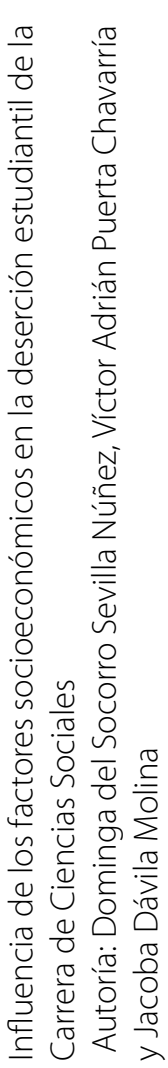 \\
\hline 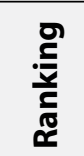 & 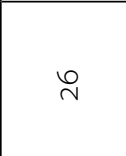 & $\stackrel{\sim}{\sim}$ & $\widehat{\searrow}$ & $\stackrel{\infty}{\sim}$ & ป & શે & in & $\bar{m}$ & $\stackrel{N}{m}$ \\
\hline
\end{tabular}




\begin{tabular}{|c|c|c|c|c|c|c|c|c|c|}
\hline $\begin{array}{l}\overline{\mathbb{0}} \\
\stackrel{0}{\circ}\end{array}$ & $\hat{n}_{\tilde{q}}^{n}$ & $\bar{b}$ & ำ & fิ & $\stackrel{\leftrightarrow}{\forall}$ & $\stackrel{\Re}{\sim}$ & $\stackrel{\text { กิ }}{\forall}$ & $\stackrel{\Sigma}{\sigma}$ & $\stackrel{\mathfrak{f}}{\underset{f}{*}}$ \\
\hline 㟔 & $\begin{array}{l}\stackrel{\circ}{N} \\
\stackrel{N}{n}\end{array}$ & $\stackrel{\stackrel{n}{n}}{\sim}$ & $\frac{b}{m}$ & $\begin{array}{l}\stackrel{v}{N} \\
m\end{array}$ & $\stackrel{\stackrel{\sim}{n}}{\sim}$ & $\stackrel{\text { in }}{\stackrel{N}{2}}$ & $\stackrel{\text { ¿ }}{\mathrm{m}}$ & $\stackrel{\infty}{\sim}$ & 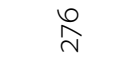 \\
\hline 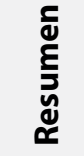 & $\stackrel{m}{\grave{n}}$ & $\begin{array}{l}\text { ○ } \\
\text { ○ }\end{array}$ & $\stackrel{m}{\sim}$ & $\underline{\bar{m}}$ & ᄋे & $\stackrel{\infty}{\sim}$ & $\stackrel{\infty}{\sim}$ & $\stackrel{m}{i}$ & $\underset{\bullet}{\bullet}$ \\
\hline 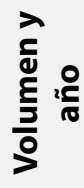 & 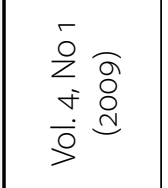 & 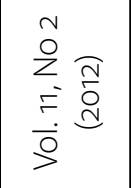 & 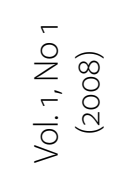 & 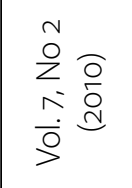 & 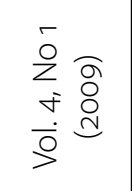 & 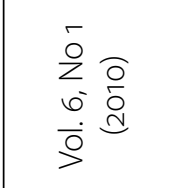 & 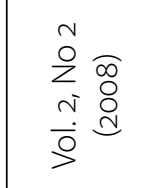 & 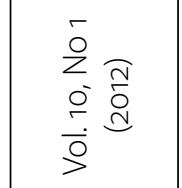 & 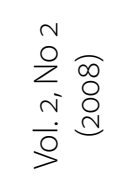 \\
\hline 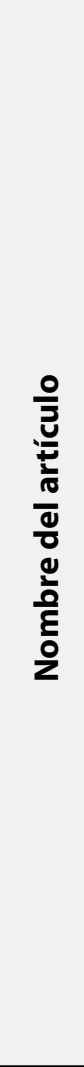 & 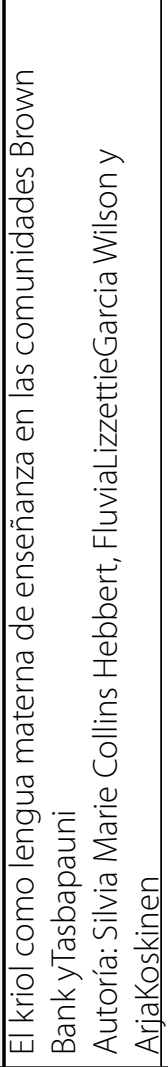 & 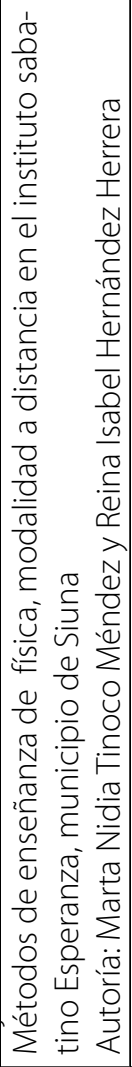 & 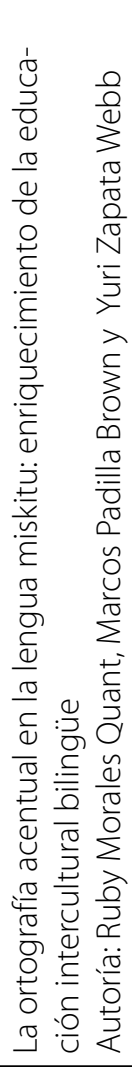 & 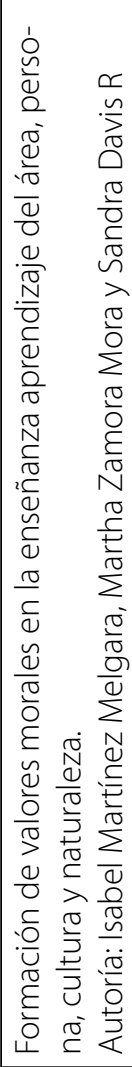 & 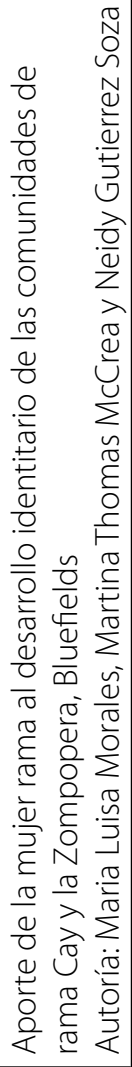 & 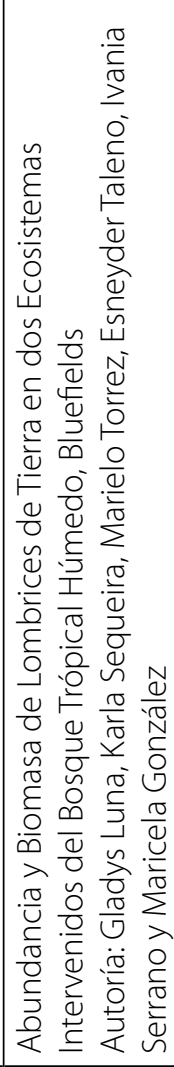 & 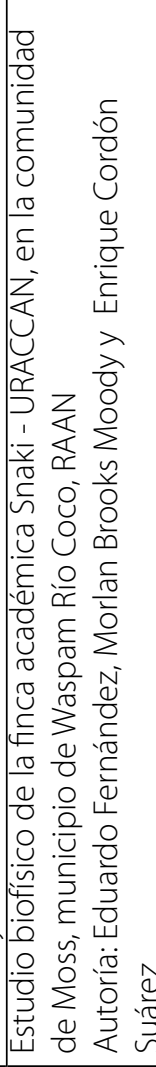 & 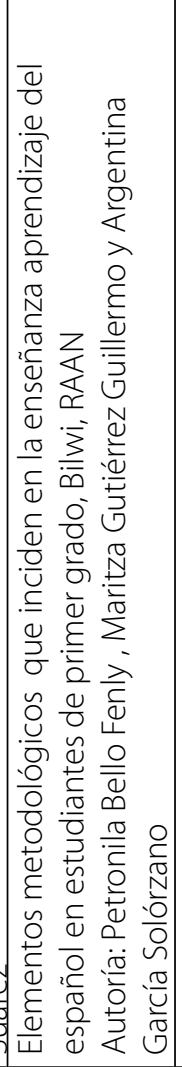 & 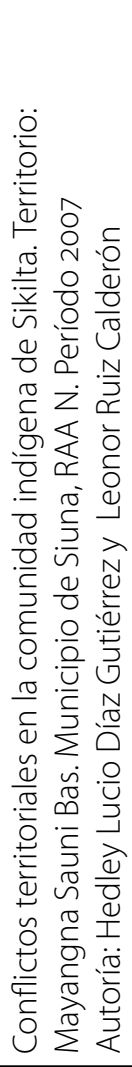 \\
\hline 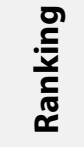 & $\stackrel{m}{m}$ & m & $\stackrel{m}{m}$ & $\stackrel{m}{m}$ & $\hat{m}$ & $\stackrel{\infty}{m}$ & ले & 守 & 广 \\
\hline
\end{tabular}




\begin{tabular}{|c|c|c|c|c|c|c|c|c|c|}
\hline $\begin{array}{l}\overline{\stackrel{0}{0}} \\
\stackrel{0}{\circ}\end{array}$ & ষ & $\hat{\tilde{\gamma}}$ & $\hat{\tilde{y}}$ & $\stackrel{\tilde{q}}{q}$ & $\stackrel{\tilde{n}}{\vartheta}$ & $\stackrel{\tilde{m}}{q}$ & $\stackrel{n}{\forall}$ & 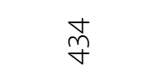 & $\stackrel{\stackrel{N}{\vartheta}}{\forall}$ \\
\hline 峉 & $\stackrel{\stackrel{ }{\sim}}{\sim}$ & 守 & 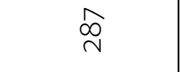 & 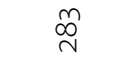 & $\stackrel{\stackrel{\sim}{\sim}}{m}$ & $\stackrel{\circ}{\curvearrowright}$ & 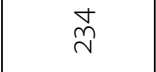 & $\stackrel{\infty}{N}$ & ㅇ \\
\hline 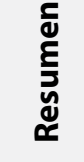 & ద̄ & $\stackrel{\text { ㅇ }}{\circ}$ & 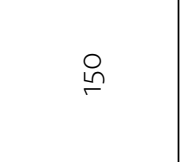 & $\underset{\sim}{\sim}$ & $\stackrel{\circ}{\rightleftharpoons}$ & $\stackrel{\leftrightarrow}{\varrho}$ & ర్ & 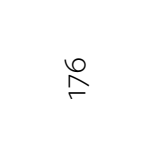 & $\stackrel{\stackrel{N}{n}}{\sim}$ \\
\hline 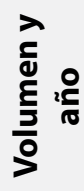 & 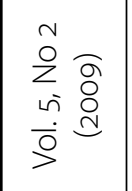 & 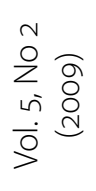 & 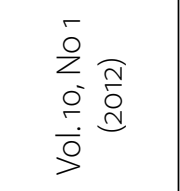 & 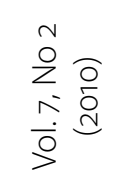 & 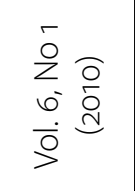 & 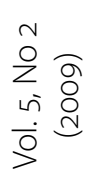 & 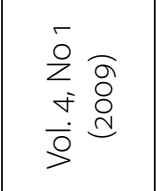 & 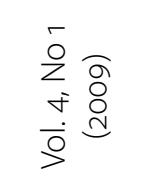 & 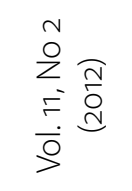 \\
\hline 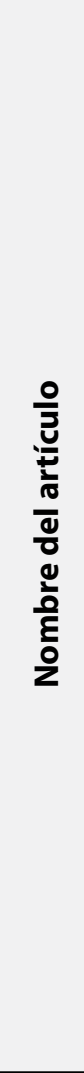 & 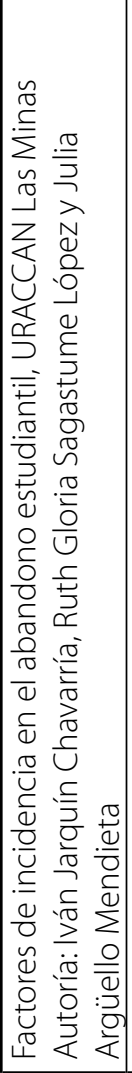 & 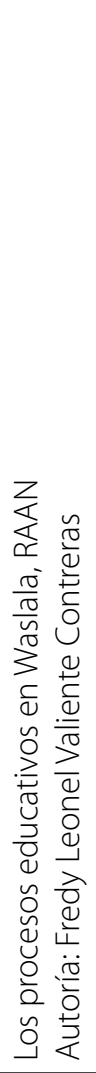 & 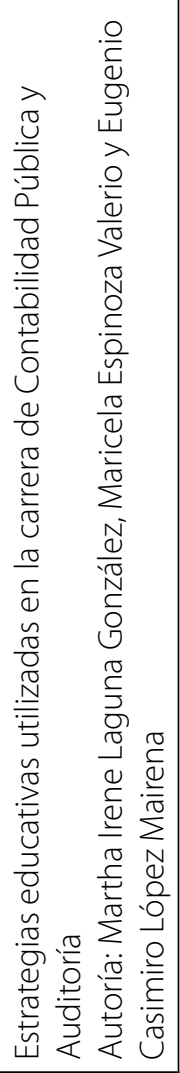 & 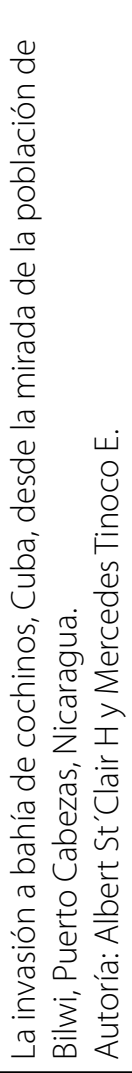 & 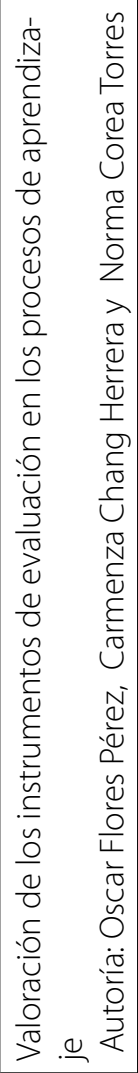 & 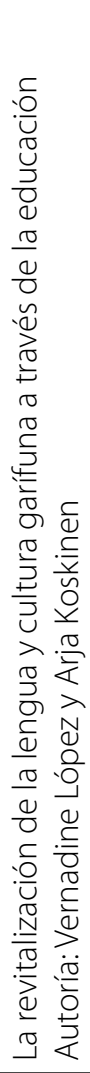 & 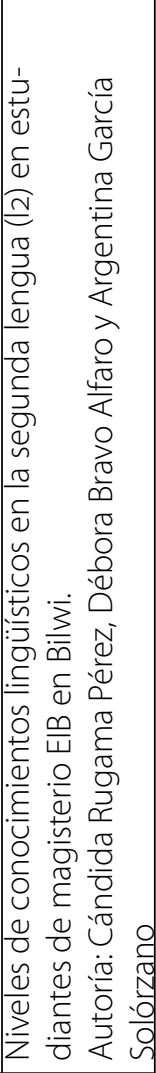 & 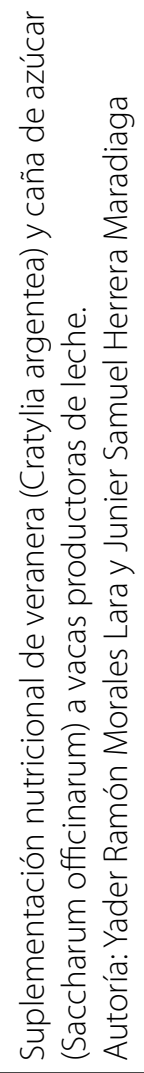 & 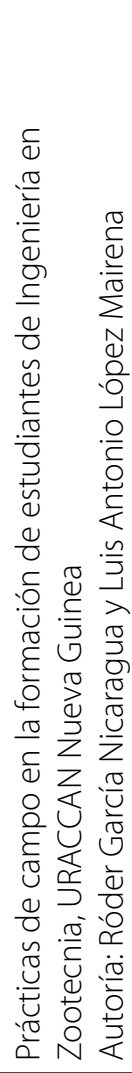 \\
\hline 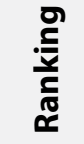 & $\stackrel{\text { f }}{\text { }}$ & $\stackrel{\text { V }}{\checkmark}$ & $\forall$ & f & q & f & $\stackrel{\infty}{\forall}$ & $\underset{\forall}{\sigma}$ & in \\
\hline
\end{tabular}


Cuadro No. 3. Ranking de 50 artículos más consultados de la Revista Caribe en el Portal de Revistas de URACCAN período junio 2011 a octubre del 2013

\begin{tabular}{|c|c|c|c|c|c|c|c|c|c|c|c|c|}
\hline 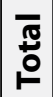 & $\underset{6}{*}$ & ڤ્مి & $\overline{\tilde{\sigma}}$ & ఓ⿵⺆一 & 点 & 诂 & $\stackrel{m}{w}$ & 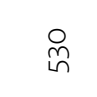 & in & $\stackrel{\infty}{i n}$ & oे & $\stackrel{n}{\gamma}$ \\
\hline tᄒㅎㅁ & $\stackrel{n}{f}$ & $\underset{\sim}{N}$ & $\underset{⿱}{\stackrel{+}{f}}$ & $\hat{\vartheta}$ & $\stackrel{\infty}{\forall}$ & $\stackrel{\substack{m \\
m}}{ }$ & $\stackrel{+}{m}$ & $\stackrel{m}{f}$ & סे & $\stackrel{\llcorner}{f}$ & ठे & $\stackrel{n}{\sim}$ \\
\hline 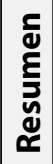 & શे & $\stackrel{\infty}{m}$ & nิ & $\stackrel{\infty}{\infty}$ & $\stackrel{\circ}{\sim}$ & $\begin{array}{l}\stackrel{\infty}{\infty} \\
\stackrel{2}{2}\end{array}$ & ô & 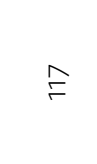 & 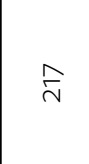 & $\stackrel{n}{n}$ & $\begin{array}{l}\infty \\
\substack{\infty \\
\sim}\end{array}$ & $\underset{m}{\stackrel{N}{m}}$ \\
\hline 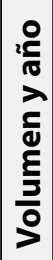 & 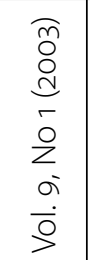 & 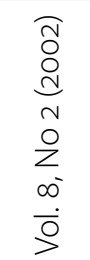 & 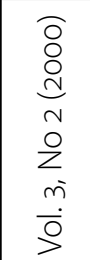 & 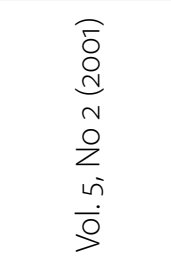 & 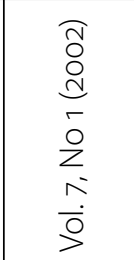 & 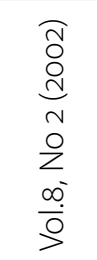 & 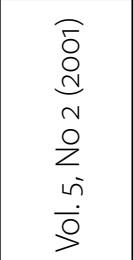 & 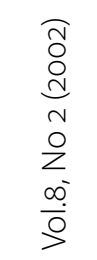 & 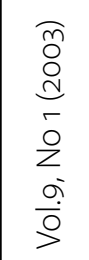 & 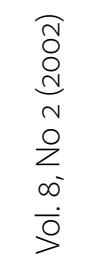 & 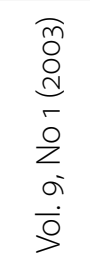 & 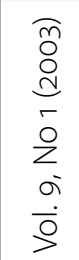 \\
\hline 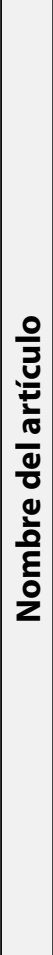 & 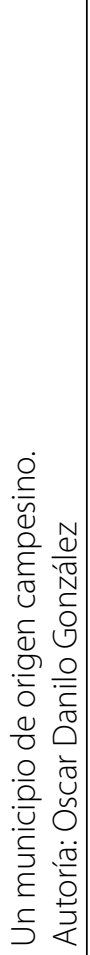 & 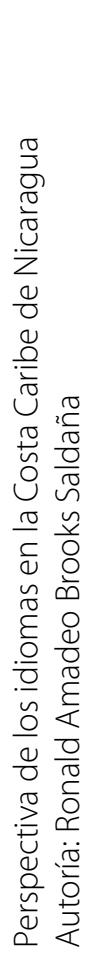 & 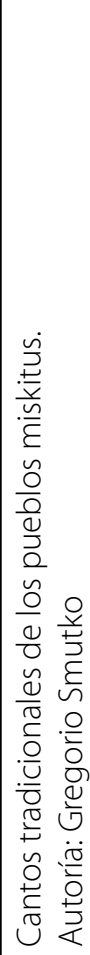 & 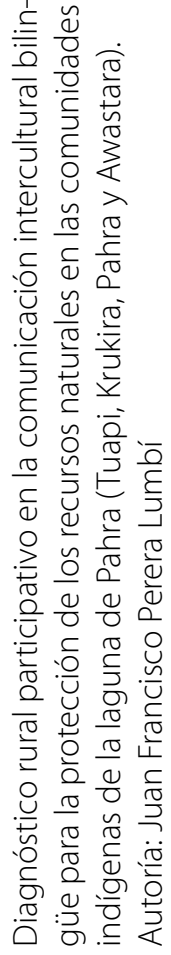 & 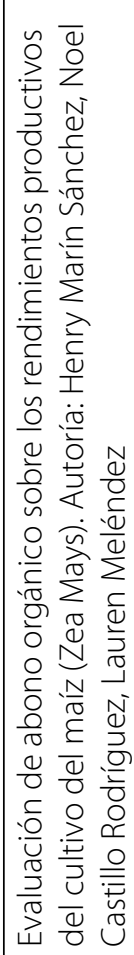 & 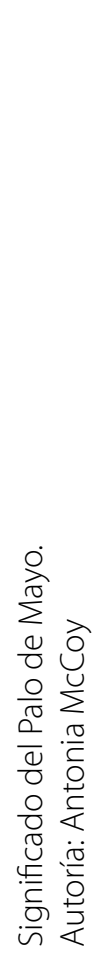 & 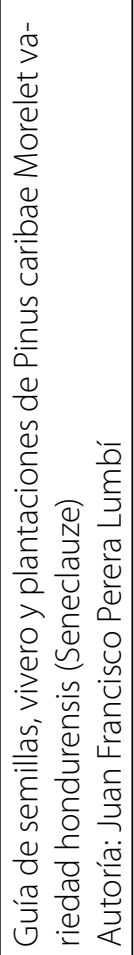 & 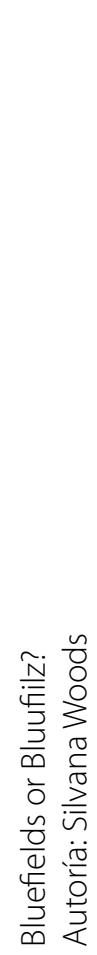 & 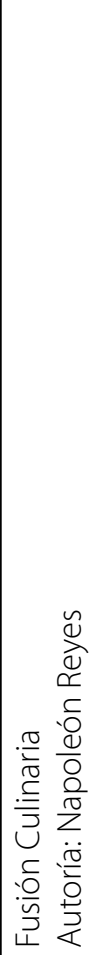 & 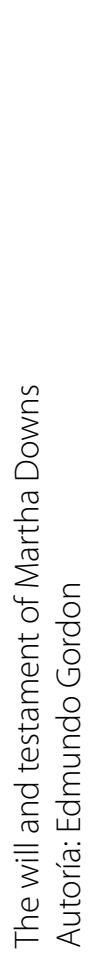 & 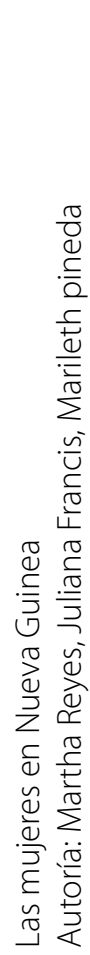 & 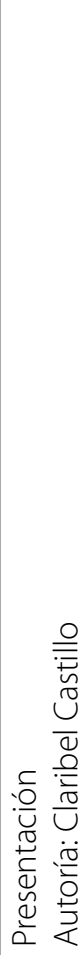 \\
\hline 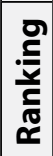 & $r$ & N & $m$ & $\nabla$ & in & 0 & $n$ & $\infty$ & $a$ & 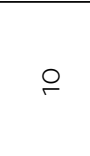 & $F$ & $\stackrel{\sim}{\sim}$ \\
\hline
\end{tabular}




\begin{tabular}{|c|c|c|c|c|c|c|c|c|c|c|c|}
\hline $\begin{array}{l}\bar{\Xi} \\
\stackrel{0}{0}\end{array}$ & $\stackrel{\hat{n}}{\sigma}$ & $\stackrel{\circ}{f}$ & $\stackrel{\circ}{\forall}$ & $\stackrel{\curvearrowright}{\text { mे }}$ & $\underset{\text { V }}{\text { V }}$ & $\stackrel{\infty}{\check{r}}$ & 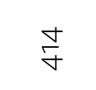 & Oे & $\begin{array}{l}\bullet \\
\stackrel{+}{+}\end{array}$ & Õ & $\stackrel{m}{\text { n}}$ \\
\hline ț & $\stackrel{\stackrel{N}{N}}{ }$ & 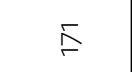 & $\stackrel{m}{\text { on }}$ & $\stackrel{\hat{n}}{\sim}$ & ô & $\stackrel{\infty}{\rightleftharpoons}$ & $\stackrel{\infty}{\infty}$ & $\stackrel{+}{\stackrel{\sim}{N}}$ & $\stackrel{+}{\stackrel{*}{N}}$ & $\stackrel{\bar{\infty}}{\sim}$ & $\begin{array}{l}\infty \\
\emptyset \\
\bullet\end{array}$ \\
\hline 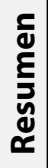 & $\stackrel{\hat{\sim}}{\sim}$ & $\stackrel{\hat{\nu}}{\hat{v}}$ & $\underline{n}$ & $\stackrel{\text { ON }}{\sim}$ & 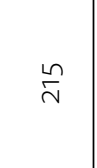 & $\stackrel{\text { ㅁ }}{\sim}$ & $\stackrel{\stackrel{\sim}{N}}{\sim}$ & 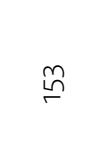 & $\stackrel{\sim}{\sim}$ & $\stackrel{\underset{\sim}{ }}{ }$ & $\stackrel{\stackrel{\sim}{N}}{\sim}$ \\
\hline 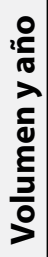 & 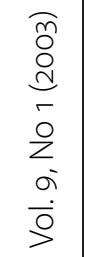 & 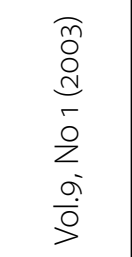 & 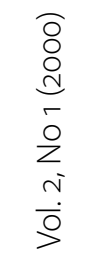 & 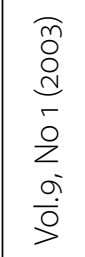 & 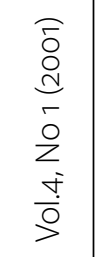 & 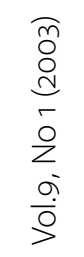 & 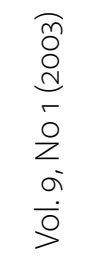 & 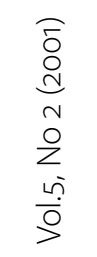 & 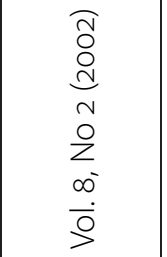 & 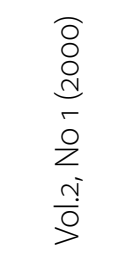 & 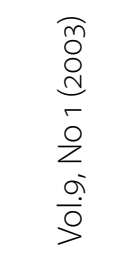 \\
\hline 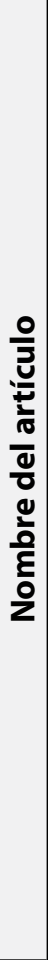 & 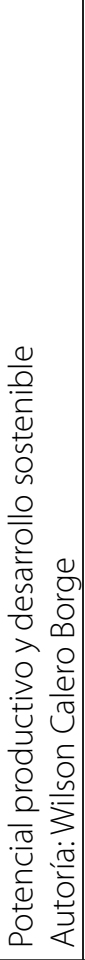 & 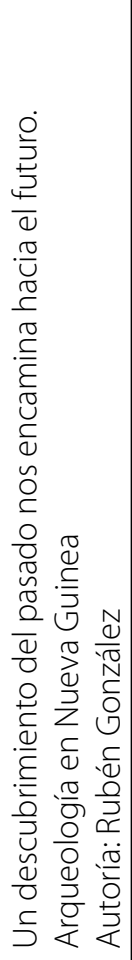 & 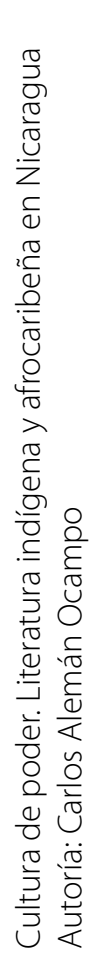 & 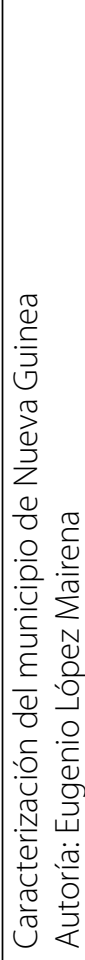 & 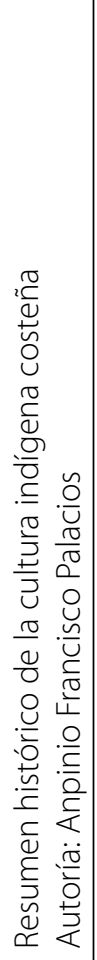 & 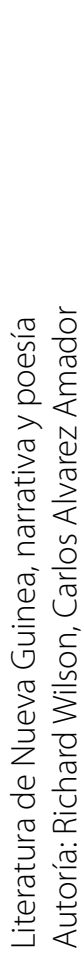 & 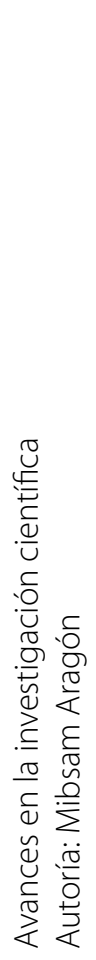 & 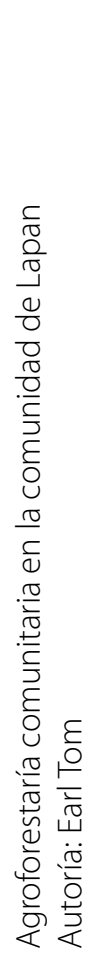 & 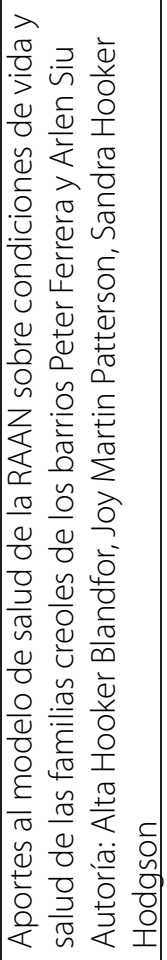 & 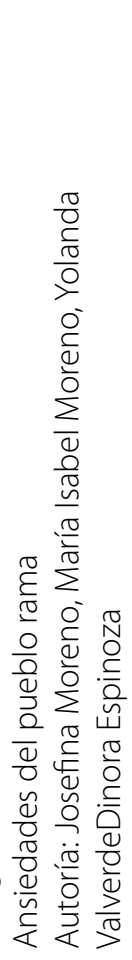 & 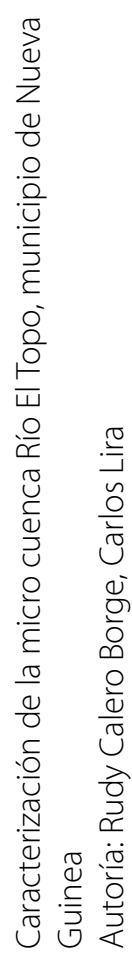 \\
\hline 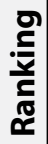 & $\stackrel{m}{r}$ & $\underset{\leftarrow}{\searrow}$ & $\stackrel{\llcorner}{\sim}$ & $\stackrel{\bullet}{r}$ & 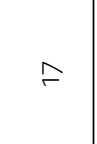 & $\stackrel{\infty}{\sim}$ & $\stackrel{\text { g }}{\ulcorner}$ & 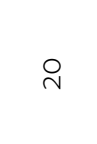 & $\bar{N}$ & $\approx$ & $\stackrel{\sim}{\sim}$ \\
\hline
\end{tabular}




\begin{tabular}{|c|c|c|c|c|c|c|c|c|c|c|}
\hline $\begin{array}{l}\bar{\Xi} \\
\stackrel{0}{\circ}\end{array}$ & $\stackrel{\text { }}{n}$ & nิ & $\stackrel{\substack{n \\
m}}{\infty}$ & $\stackrel{\bar{m}}{m}$ & 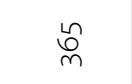 & $\stackrel{\infty}{\stackrel{\infty}{n}}$ & $\stackrel{\stackrel{\omega}{n}}{n}$ & $\stackrel{\infty}{m}$ & $\underset{m}{\forall}$ & $\stackrel{\text { mे }}{m}$ \\
\hline 눔 & $\underset{\forall}{\stackrel{\Xi}{\Xi}}$ & $\underset{\Sigma}{ }$ & $\underset{\sigma}{\widetilde{\sigma}}$ & 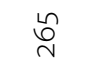 & $\stackrel{\grave{n}}{ }$ & $\stackrel{\sim}{\sim}$ & $\stackrel{m}{\sim}$ & $\stackrel{\circ}{\stackrel{\leftrightarrow}{N}}$ & $\hat{\vartheta}$ & $\stackrel{+}{\sim}$ \\
\hline 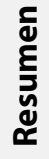 & $\stackrel{\text { ㄱ }}{\mathrm{O}}$ & $\stackrel{\bullet}{\stackrel{0}{N}}$ & $\stackrel{\sim}{\sim}$ & $\stackrel{\varphi}{\rightleftharpoons}$ & $\stackrel{\infty}{\sim}$ & $\underset{\sim}{\stackrel{\Xi}{c}}$ & $\stackrel{N}{\sim}$ & $\stackrel{\infty}{\sigma}$ & $\curvearrowright$ & $\stackrel{\stackrel{\sim}{\sim}}{\sim}$ \\
\hline 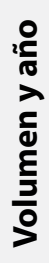 & 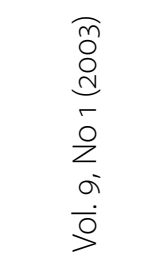 & 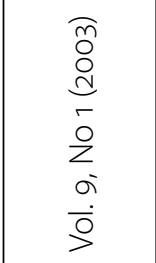 & 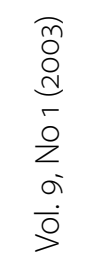 & 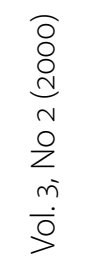 & 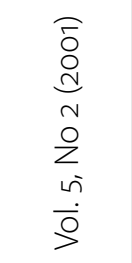 & 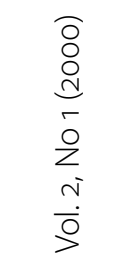 & 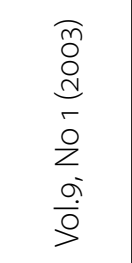 & 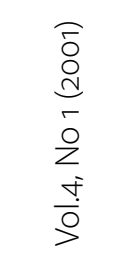 & 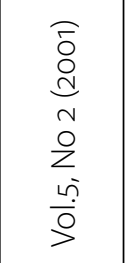 & 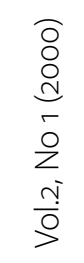 \\
\hline 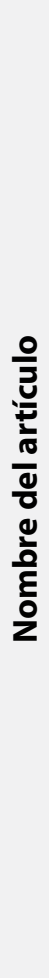 & 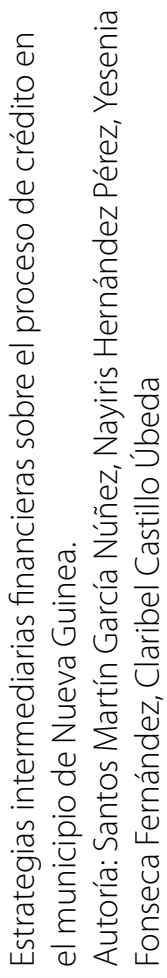 & 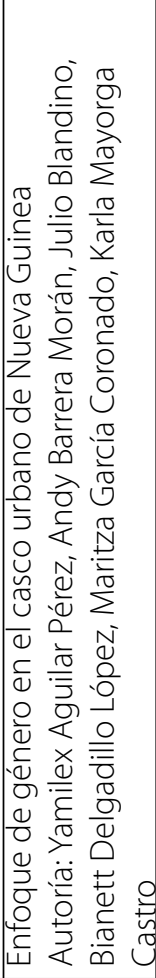 & 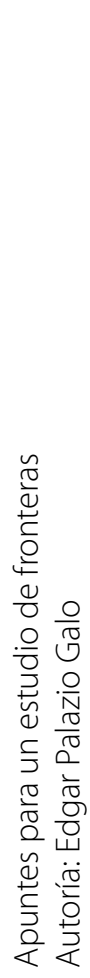 & 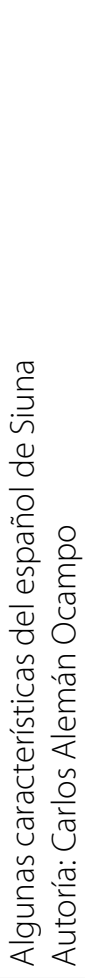 & 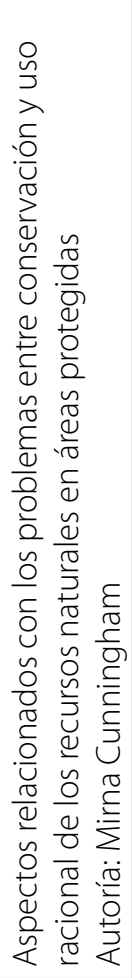 & 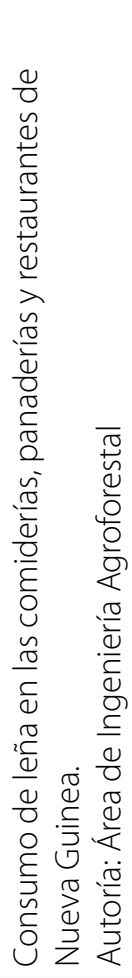 & 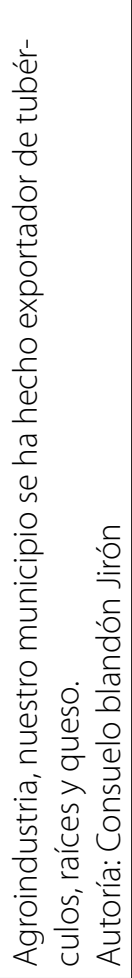 & 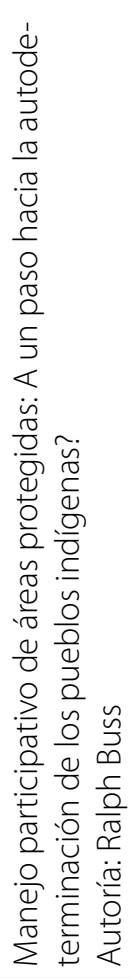 & 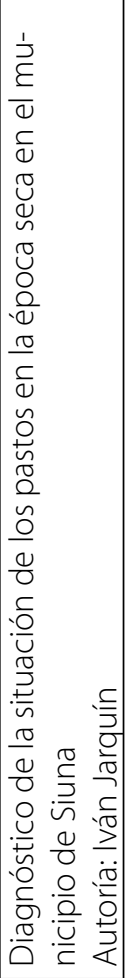 & 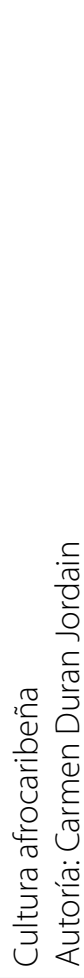 \\
\hline$\frac{\text { o }}{\frac{5}{x}}$ & $\stackrel{\forall}{\sim}$ & $\stackrel{\llcorner}{\sim}$ & $\stackrel{\bullet}{v}$ & $\widehat{৩}$ & $\stackrel{\infty}{\sim}$ & ஓે & $\stackrel{\mathrm{m}}{ }$ & $\bar{m}$ & $\stackrel{N}{m}$ & $\stackrel{m}{m}$ \\
\hline
\end{tabular}




\begin{tabular}{|c|c|c|c|c|c|c|c|c|c|c|c|}
\hline $\begin{array}{l}\bar{\Xi} \\
\stackrel{0}{0}\end{array}$ & $\stackrel{\infty}{m}$ & $\stackrel{\stackrel{m}{m}}{m}$ & 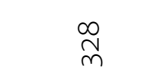 & $\underset{\mathrm{n}}{\mathrm{N}}$ & $\underset{\mathrm{N}}{\mathrm{N}}$ & $\underset{\sim}{N}$ & $\stackrel{\text { N }}{m}$ & $\frac{a}{m}$ & $\stackrel{\llcorner}{m}$ & ర్ & ㅇ \\
\hline$\frac{\pi}{0}$ & $\stackrel{\bullet}{N}$ & $\stackrel{\stackrel{L}{0}}{\stackrel{\nu}{v}}$ & $\stackrel{\text { ㅁ }}{\sim}$ & $\underset{\leftarrow}{\stackrel{m}{\leftarrow}}$ & $\stackrel{\stackrel{\sim}{N}}{N}$ & $\overline{\bar{N}}$ & $\stackrel{\infty}{\sim}$ & $\stackrel{\text { V }}{v}$ & ᄋ & 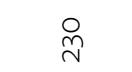 & $\stackrel{m}{\grave{n}}$ \\
\hline 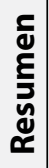 & $\stackrel{ }{\rightleftharpoons}$ & ○ & ๑ & $\stackrel{\curvearrowright}{\curvearrowright}$ & ลे & $\underset{\rightleftharpoons}{\mp}$ & $\infty$ & 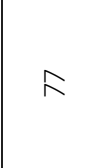 & $\stackrel{\stackrel{\sim}{\sim}}{\sim}$ & $\Sigma$ & $\infty$ \\
\hline 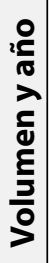 & 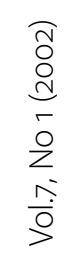 & 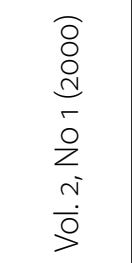 & 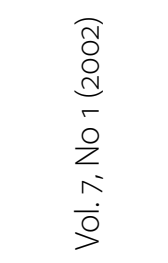 & 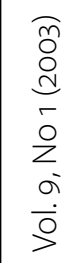 & 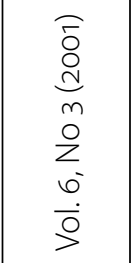 & 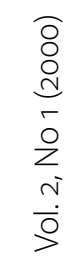 & 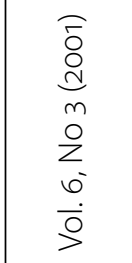 & 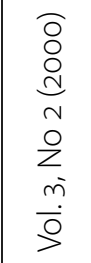 & 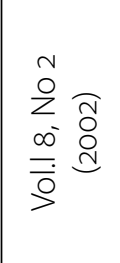 & 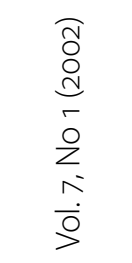 & 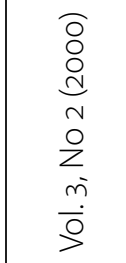 \\
\hline 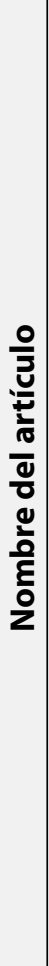 & 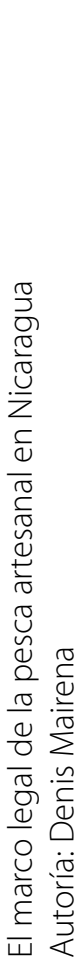 & 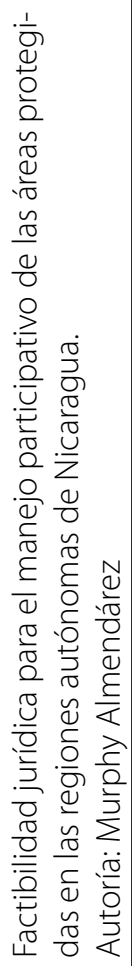 & 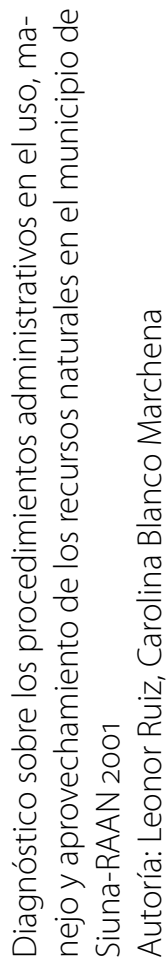 & 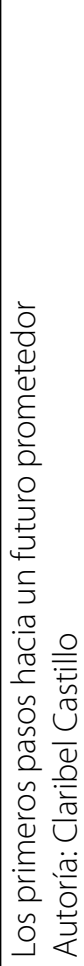 & 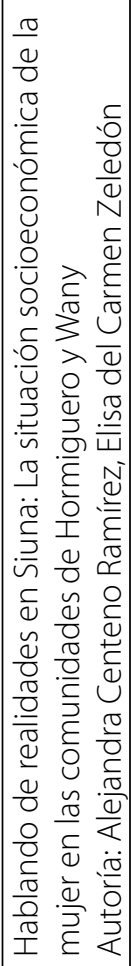 & 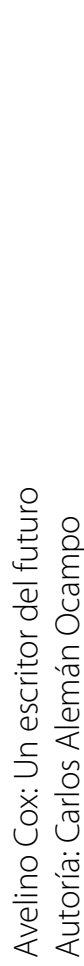 & 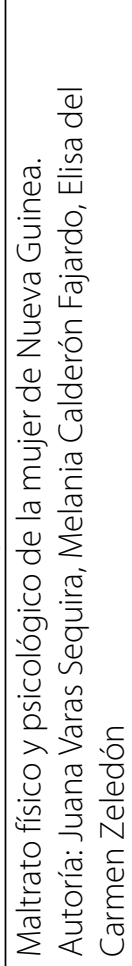 & 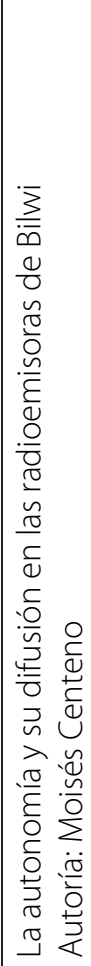 & 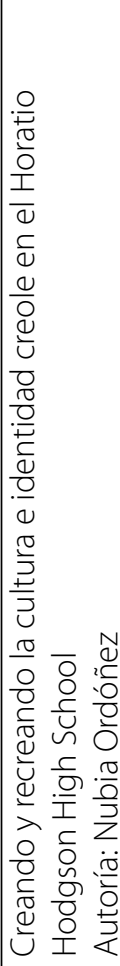 & 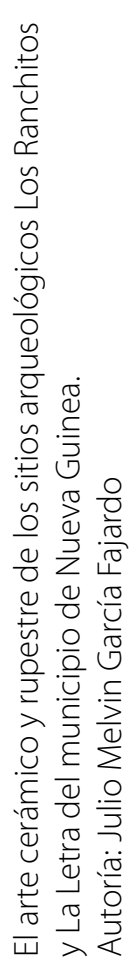 & 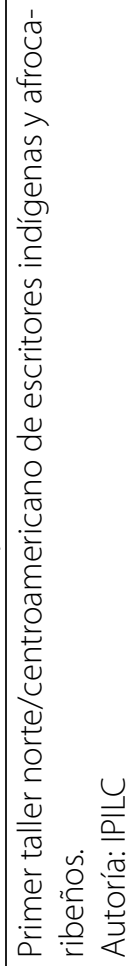 \\
\hline 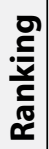 & $\stackrel{+}{m}$ & $\stackrel{\llcorner}{m}$ & $\stackrel{\bullet}{m}$ & $\hat{m}$ & $\stackrel{\infty}{m}$ & ळे & $\stackrel{\circ}{+}$ & $\bar{\gamma}$ & ঙั & $\stackrel{m}{\forall}$ & $\underset{\forall}{\forall}$ \\
\hline
\end{tabular}




\begin{tabular}{|c|c|c|c|c|c|c|}
\hline ָే & ๙ิ & $\begin{array}{l}\text { @े } \\
\text { ণे }\end{array}$ & ๙ิ & 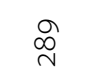 & $\begin{array}{l}\infty \\
\stackrel{\infty}{\sim}\end{array}$ & $\stackrel{\mathscr{L}_{N}}{\stackrel{N}{n}}$ \\
\hline$\frac{4}{0}$ & $\stackrel{\llcorner}{\grave{N}}$ & તี & $\underset{\infty}{\check{\infty}}$ & $\underset{\sim}{\sim}$ & $\underset{\sim}{\infty}$ & $\stackrel{\sim}{\sim}$ \\
\hline 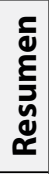 & $\infty$ & $\stackrel{N}{\sim}$ & 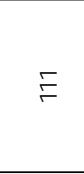 & $\stackrel{m}{\sqcup}$ & $\stackrel{\text { ㅁ }}{\sim}$ & $\stackrel{m}{r}$ \\
\hline 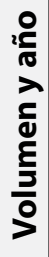 & 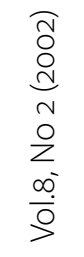 & 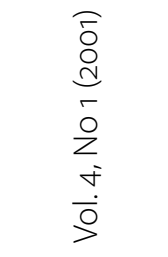 & 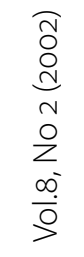 & 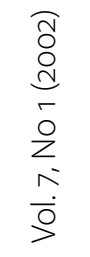 & 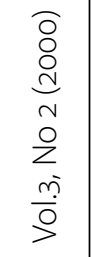 & 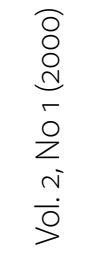 \\
\hline 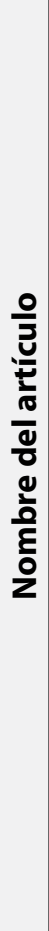 & 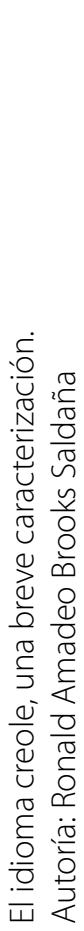 & 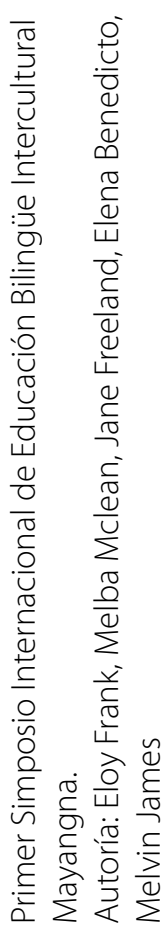 & 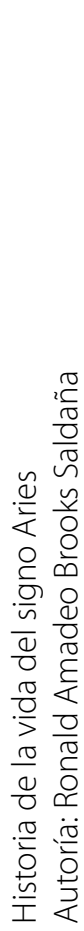 & 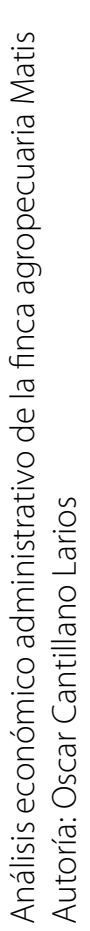 & 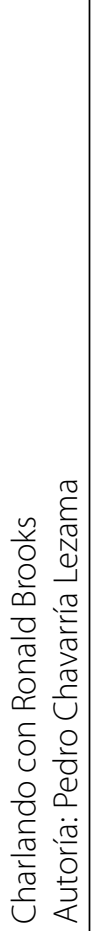 & 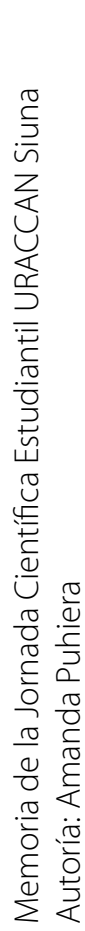 \\
\hline 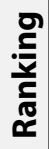 & $\stackrel{\llcorner}{\sigma}$ & $\stackrel{b}{q}$ & f & $\stackrel{\infty}{\underset{\sim}{*}}$ & g̊ & in \\
\hline
\end{tabular}




\section{Revista Ciencia e Interculturalidad en el Portal de Revistas de Nicaragua}

En el primer monitoreo realizado al Portal de Revistas de Nicaragua, encontramos que en el período de 2012 a noviembre del 2013, la Revista Ciencia e Interculturalidad recibió un total de 12,522 visitas de revisión a resúmenes y 13,881 para revisar o descargar artículo completo en PDF. A continuación se presenta el ranking de los artículos más leídos en este portal nacional.

Cuadro No. 4. Ranking de 10 artículos más leído de Ciencia e Interculturalidad en el Portal de Revistas de Nicaragua

\begin{tabular}{|c|c|c|c|c|}
\hline \multirow{2}{*}{ Ranking } & \multirow{2}{*}{ Título } & \multicolumn{3}{|c|}{ Consulta } \\
\hline & & Resumen & Pdf & Total \\
\hline 1 & $\begin{array}{l}\text { Influencia de las fases lunares sobre el rendimiento del } \\
\text { maíz (Zea mays variedad NB6), Vol. 10, (2012) } \\
\text { Larry Flores Martínez, Félix Meléndez Mejía, Gladys } \\
\text { Luna Bello y Eliseo González Lazo }\end{array}$ & 297 & 727 & 1024 \\
\hline 2 & $\begin{array}{l}10 \text { años de historia fundacional de la URACCAN Siuna, } \\
\text { Las Minas, Vol. 10, \# } 1 \text { (2012) } \\
\text { Fredy Leonel Valiente Contreras y Víctor Manuel del Cid } \\
\text { Lucero }\end{array}$ & 220 & 262 & 482 \\
\hline 3 & $\begin{array}{l}\text { Elementos metodológicos que inciden en la enseñan- } \\
\text { za aprendizaje del español en estudiantes de primer } \\
\text { grado, Bilwi, RAAN, Vol. 10, } 2012 \\
\text { Petronila Bello Fenly, Maritza Gutiérrez Guillermo y } \\
\text { Argentina García Solórzano }\end{array}$ & 213 & 248 & 461 \\
\hline 4 & $\begin{array}{l}\text { Estrategias educativas utilizadas en la carrera de } \\
\text { Contabilidad Pública y Auditoría,Vol. 10, (2012) } \\
\text { Martha Irene Laguna González, Maricela Espinoza } \\
\text { Valerio y Eugenio Casimiro López Mairena }\end{array}$ & 203 & 225 & 428 \\
\hline 5 & $\begin{array}{l}\text { La desintegración familiar y el aprendizaje en niñas y } \\
\text { niños de cuarto grado, Vol. 1, (2008) } \\
\text { María Estela Vargas Tess, Nalia Rosales Cunningham y } \\
\text { Argentina García Solórzano }\end{array}$ & 173 & 213 & 386 \\
\hline 6 & $\begin{array}{l}\text { Acceso y permanencia a la Educación Superior de mu- } \\
\text { jeres indígenas Mayangnas, URACCAN Las Minas, } 2009 \\
\text { - 2010, Vol. 10, (2012) } \\
\text { Sorayda del Carmen Herrera Siles }\end{array}$ & 198 & 164 & 362 \\
\hline 7 & $\begin{array}{l}\text { Las repúblicas bolivariana de Venezuela y } \\
\text { Nicaragua, en el marco del acuerdo energético", Vol. } \\
\text { 11, (2012) } \\
\text { Dani José Villalobos Soto }\end{array}$ & 242 & 113 & 355 \\
\hline
\end{tabular}




\begin{tabular}{|c|l|c|c|c|}
\hline \multirow{2}{*}{ Ranking } & \multicolumn{1}{|c|}{ Título } & \multicolumn{1}{|c|}{ Consulta } \\
\cline { 3 - 5 } 8 & \multicolumn{1}{|c|}{ Resumen } & \multicolumn{1}{|c|}{ Pdf } & Total \\
\hline \multirow{2}{*}{9} & $\begin{array}{l}\text { Eficiencia Terminal en programas de licenciatura, } \\
\text { URACCAN Bilwi 1995 -2005, Vol. 10, (2012) } \\
\text { Argentina García Solórzano yEarl Tom }\end{array}$ & 190 & 164 & 354 \\
\hline 10 & $\begin{array}{l}\text { Venezuela en el proceso de incorporación al } \\
\text { MERCOSUR, Vol. 10, (2012) } \\
\text { Dani José Villalobos Soto y Enio Enrique Ortiz } \\
\text { Valenzuela }\end{array}$ & 160 & 173 & 333 \\
\hline & $\begin{array}{l}\text { Participación de las mujeres en las unidades producti- } \\
\text { vas familiares rurales, Rosita, RAAN, Vol. 10, (2012) } \\
\text { Sonia del Carmen García Aguilar y Argentina García } \\
\text { Solórzano }\end{array}$ & 151 & 179 & 330 \\
\hline
\end{tabular}

\section{Revista Ciencia e Interculturalidad en Latin American Jornal Online}

A finales de junio de 2012, Latin American Jornal Online publicó su primer boletín informativo, en él se destaca que se contaba con 50 números y 385 artículos. Todos ellos estaban disponibles en su versión con texto íntegro. El número total de visitas a los artículos de agosto de 2010 a junio de 2012, fue de 44.344 artículos. La revista con mayor número de consultas fue Ceiba, con 7.811; le siguió Encuentro, con 6.774, y Ciencia e Interculturalidad, con 5.079. El número de consultas de artículos de una revista especializada se ve influenciado por el número de artículos que están disponibles en el sitio web. Algunas revistas tienen muchos más artículos que otras, lo que explica que estas revistas tengan un número mayor de consultas de artículos. En el cuadro siguiente se presenta la lista de los 10 artículos más consultados, entre ellos se encuentran dos de Ciencia e Interculturalidad (INASP, 2012).

Cuadro No. 5. Lista de los 10 artículos de diversas revistas, más consultados en LAMJOL, período agosto de 2010 a junio de 2012

\begin{tabular}{|c|c|}
\hline \multicolumn{2}{|c|}{ Listado de los 10 artículos más consultados entre agosto de 2010 y junio de 2012} \\
\hline $\begin{array}{l}\text { SY Gutiérrez, EC López. (2010) Enseñanza de la geometría en } \\
\text { Segundo año de educación secundaria bajo el enfoque de } \\
\text { competencias. Ciencia e Interculturalidad }\end{array}$ & $\begin{array}{l}\text { J Ruiz (2009) Ecología de dispersión y reclutamiento de bosques } \\
\text { húmedos tropicales. Wani } \\
\text { http://www.lamjol.info/index.php/WANI/article/view/256 }\end{array}$ \\
\hline http://www.lamjol.info/index.php/RCl/article/view/285 & $\begin{array}{l}\text { M Künne, M Strecker (2008) Arte Rupestre de México Oriental y de } \\
\text { Centro América. Wani }\end{array}$ \\
\hline $\begin{array}{l}\text { factores socioeconómicos en la deserción estudiantil de la Carrera } \\
\text { de ciencias sociales. Ciencia e Interculturalidad }\end{array}$ & http://www.lamjol.info/index.php/WANI/article/view/219 \\
\hline http://www.lamjol.info/index.php/RCl/article/view/282 & $\begin{array}{l}\text { (2006) Resúmenes de Tesis de Zootecnia, 2006. Ceiba } \\
\text { http://www.lamjol.info/index.php/CEIBA/article/view/451 }\end{array}$ \\
\hline $\begin{array}{l}\text { (2008) Resúmenes de Tesis de Zootecnia 2007. Ceiba } \\
\text { http://www.lamjol.info/index.php/CEIBA/article/view/296 }\end{array}$ & $\begin{array}{l}\text { JR Marcia (2009) Evaluación de siete híbridos de chile jalapeño } \\
\text { (Capsicum annuum). FHIA. Programa de Hortalizas }\end{array}$ \\
\hline (2008) Resúmenes de Tesis de Fitotecnia, 2007. Ceiba & http://www.lamjol.info/index.php/FHIAPH/article/view/232 \\
\hline $\begin{array}{l}\text { http://www.lamjol.info/index.php/CEIBA/article/view/295 } \\
\text { (2008) Resúmenes de Tesis de Zootecnia 2008. Ceiba }\end{array}$ & $\begin{array}{l}\text { A Barbeyto (2010) El proceso de paz en Centroamérica y el } \\
\text { reconocimiento de la diversidad étnico-cultural: El caso de } \\
\text { Nicaragua y Guatemala. Wani }\end{array}$ \\
\hline http://www.lamjol.info/index.php/CEIBA/article/view/298 & http://www.lamjol.info/index.php/WANI/article/view/264 \\
\hline
\end{tabular}

Fuente: INASP, 2012. 


\section{Consultas a LAMJOL período marzo del 2011 a octubre del 2013}

En el monitoreo realizado al Portal de en Latin American Jornal Online (LAMJOL), en el período de marzo del 2011 a octubre del 2013, la Revista Ciencia e Interculturalidad recibió un total de 53,757 revisiones de sus artículos. Las consultas trimestrales más altas se dieron en el primero y segundo trimestre del 2013 con 9,787 y 9,813 respectivamente (ver cuadro 6 y figura 2). En el cuadro 7 se presenta el ranking de los artículos más leídos en este portal nacional.

Cuadro No. 6. Consultas trimestrales y acumuladas de la Revista Ciencia e Interculturalidad en Latin American Jornal online (LAMJOL), período marzo de 2011 a octubre de 2013

\begin{tabular}{|l|c|c|c|c|c|c|c|c|r|r|r|}
\hline & \multicolumn{4}{|c|}{2011} & \multicolumn{3}{c|}{2012} & \multicolumn{3}{c|}{2013} \\
\hline Descripción & Mar & June & Oct & Dec & Mar & June & Sept & Dec & March & June & Oct \\
\hline $\begin{array}{l}\text { Total acumu- } \\
\text { lado }\end{array}$ & 1,906 & 5,158 & 7,812 & 10,335 & 13,953 & 19,152 & 23,492 & 29,700 & 39,487 & 49,300 & 53,757 \\
\hline Por trimestre & 1,906 & 3,252 & 2,654 & 2,523 & 3,618 & 5,199 & 4,340 & 6,208 & 9,787 & 9,813 & 4,457 \\
\hline
\end{tabular}

Fuente: (Sioux Cumming, 2013).

\section{Article Views by Quarter}

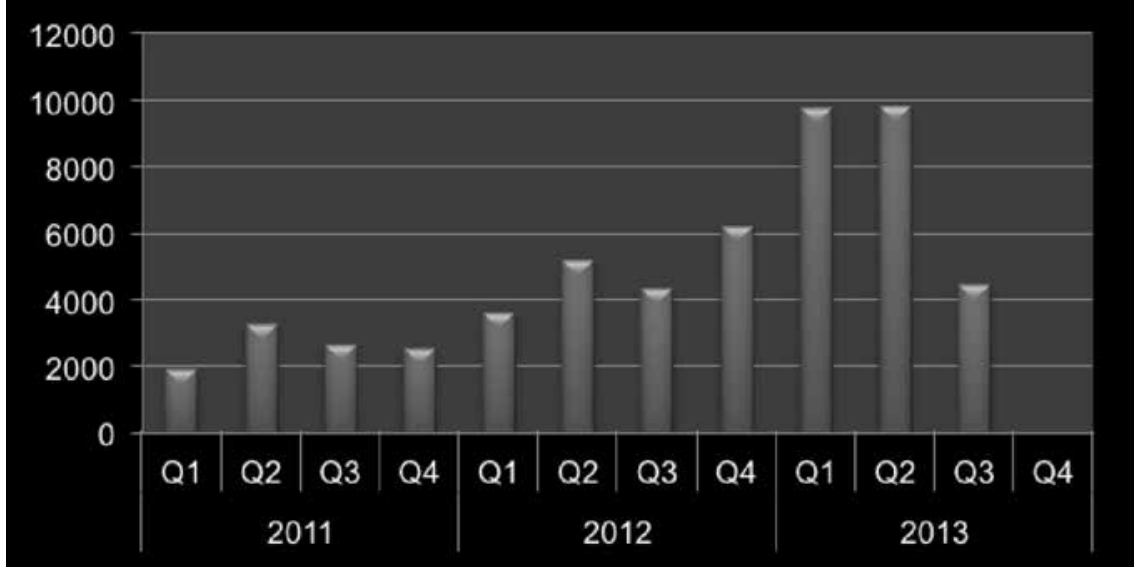

Figura No. 2. Número de visitas por trimestre de la Revista Ciencia e Interculturalidad en Latin American Jornal online (LAMJOL), período marzo de 2011 a octubre de 2013 (Sioux Cumming, 2013). 
Cuadro No. 7. Ranking de 50 artículos más consultados de la Revista ciencia e Interculturalidad en Latin American Jornal online (LAMJOL), período marzo de 2011 a octubre de 2013

\begin{tabular}{|c|c|c|c|c|c|c|c|c|c|c|c|}
\hline 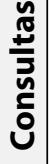 & $\begin{array}{l}\text { N } \\
\\
n\end{array}$ & $\begin{array}{l}\stackrel{8}{0} \\
\dot{0} \\
\text { m }\end{array}$ & $\stackrel{\infty}{\stackrel{\sim}{\sim}}$ & 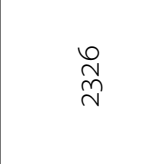 & $\stackrel{\substack{q \\
m}}{r}$ & $\underset{\xi}{\stackrel{\Sigma}{\xi}}$ & $\stackrel{\stackrel{L}{n}}{\rightleftharpoons}$ & $\underset{F}{\stackrel{F}{F}}$ & $\begin{array}{l}\stackrel{\circ}{\rightleftharpoons} \\
\stackrel{F}{F}\end{array}$ & ณू & ָે \\
\hline & 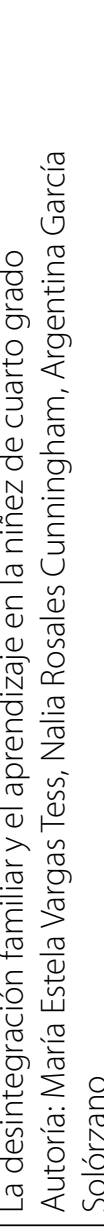 & 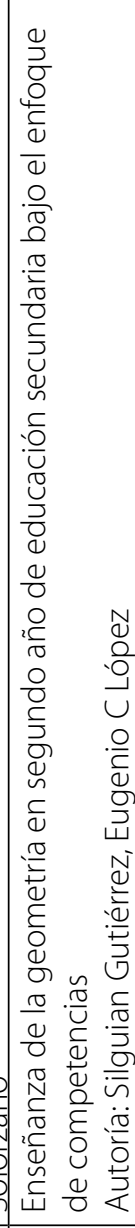 & 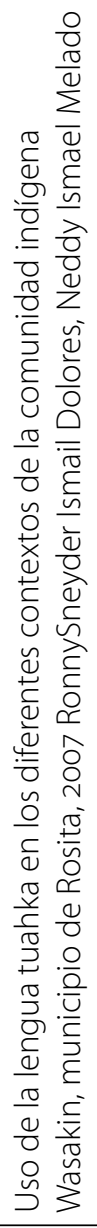 & 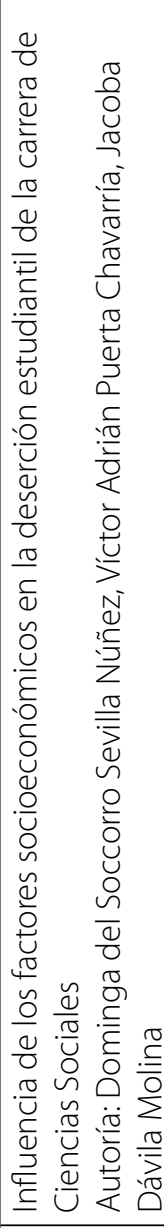 & 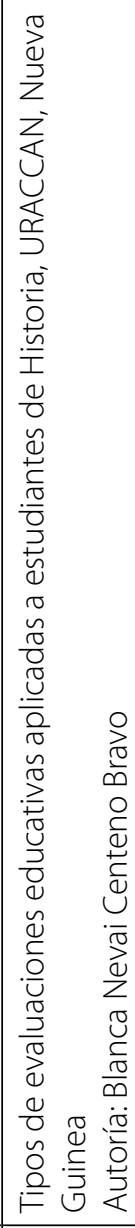 & 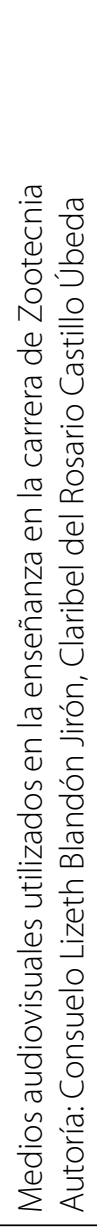 & 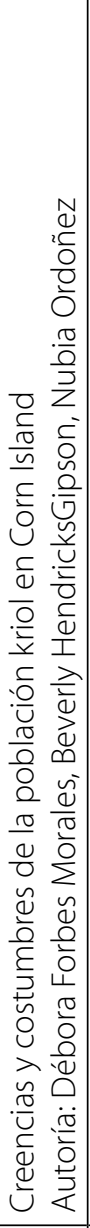 & 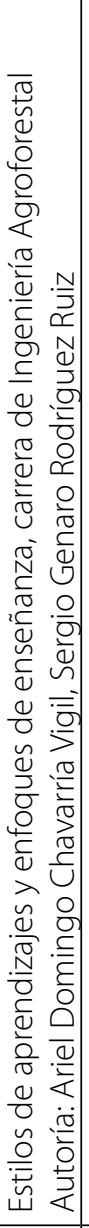 & 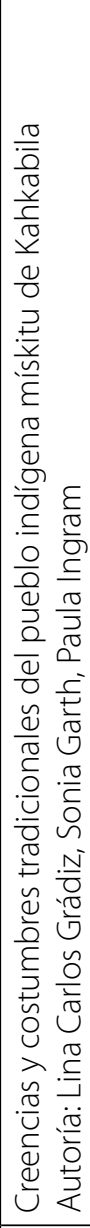 & 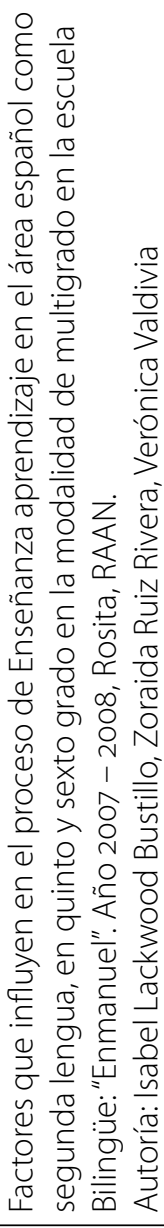 & 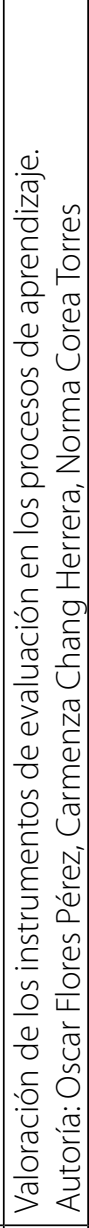 \\
\hline $\begin{array}{l}\frac{5}{\varrho} \\
\frac{\varepsilon}{5} \\
\frac{0}{\rho}\end{array}$ & 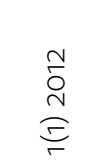 & 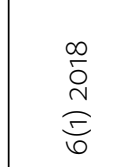 & $\underset{\substack{d \\
\stackrel{d}{d}}}{d}$ & 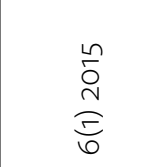 & 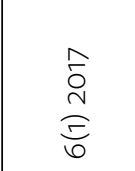 & $\frac{\stackrel{n}{o}}{\underset{i}{d}}$ & 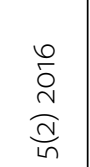 & $\frac{\stackrel{+}{D}}{\stackrel{i}{N}}$ & $\frac{\tilde{o}}{\stackrel{i}{N}}$ & $\begin{array}{l}\frac{b}{\delta} \\
\substack{d \\
\text { d }}\end{array}$ & 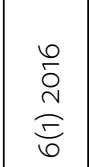 \\
\hline 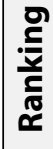 & - & $N$ & $m$ & $\nabla$ & in & 6 & $\wedge$ & $\infty$ & $a$ & $\stackrel{\circ}{\circ}$ & $F$ \\
\hline
\end{tabular}




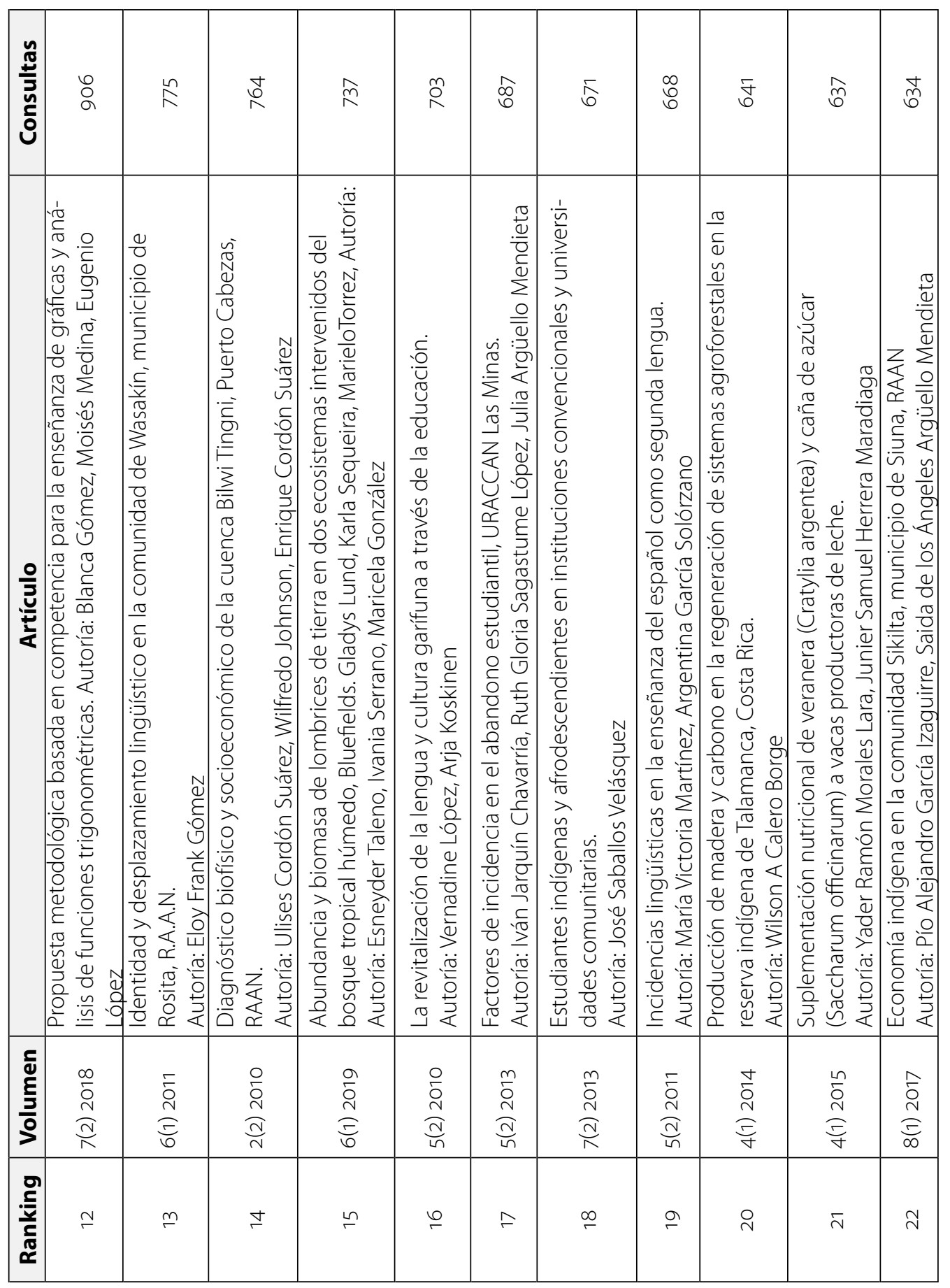




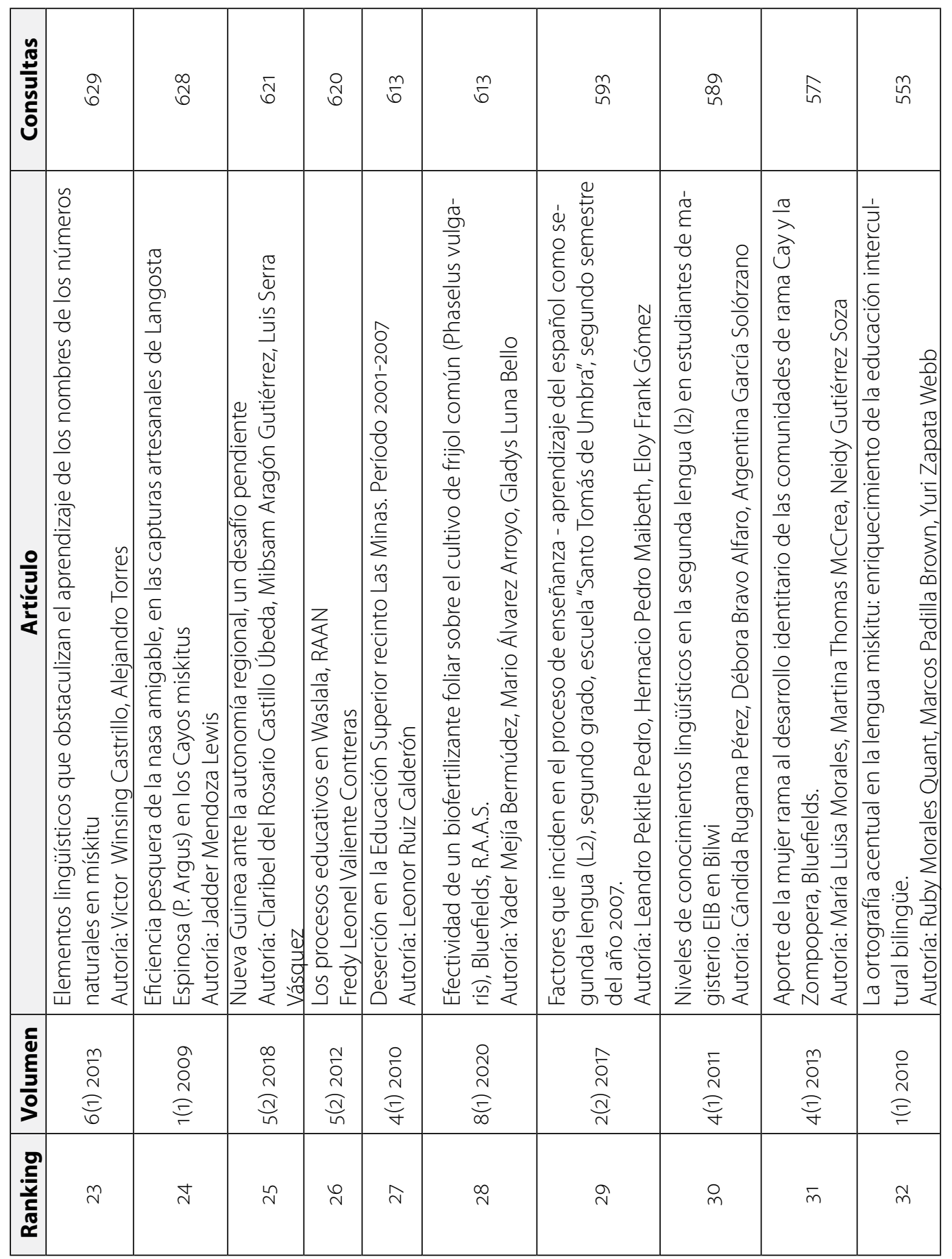




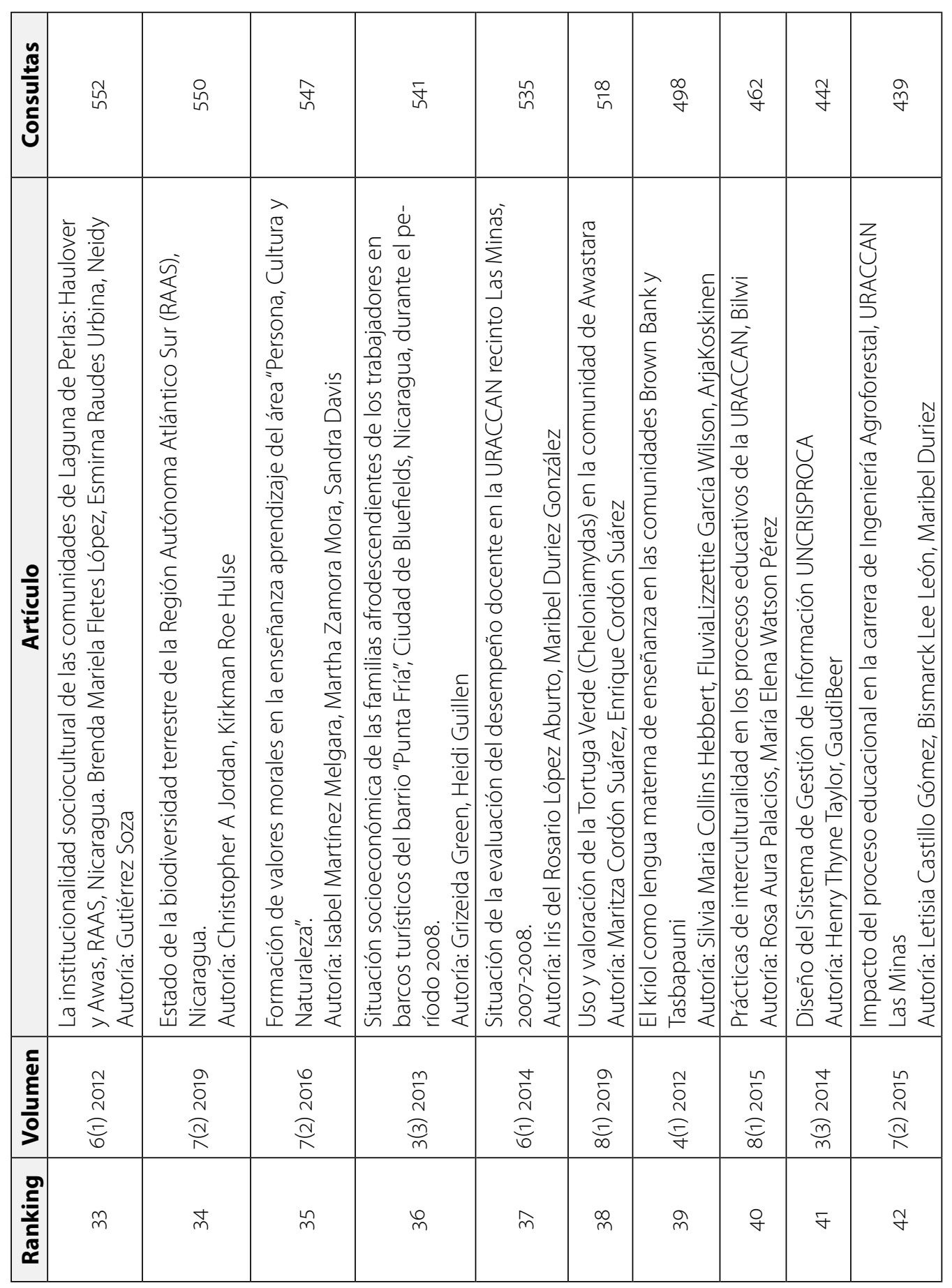




\begin{tabular}{|c|c|c|c|c|c|c|c|c|}
\hline 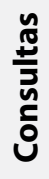 & $\stackrel{m}{\vartheta}$ & $\stackrel{m}{\vartheta}$ & $\stackrel{\text { }}{\forall}$ & $\stackrel{\bar{m}}{\forall}$ & 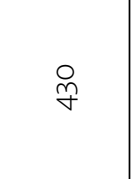 & ָे & ָิ & fे \\
\hline$\frac{\text { 을 }}{\text { 는 }}$ & 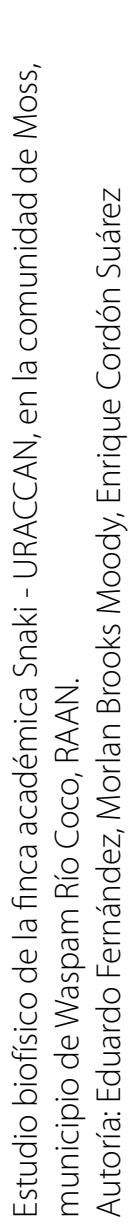 & 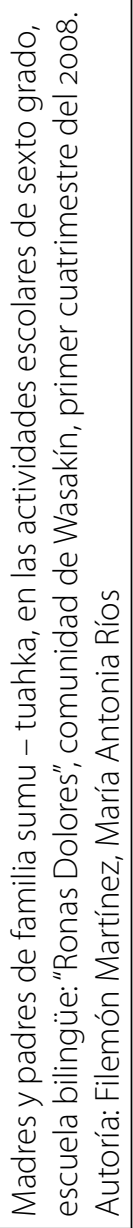 & 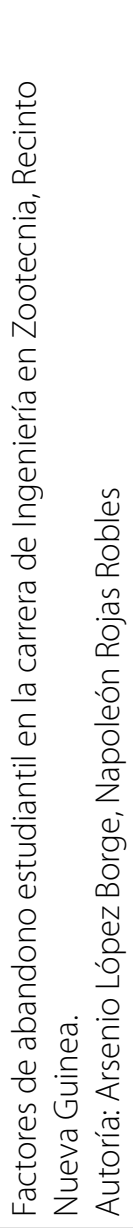 & 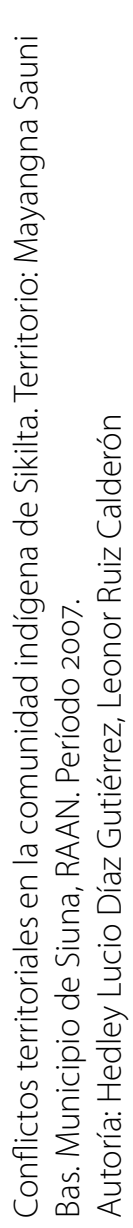 & 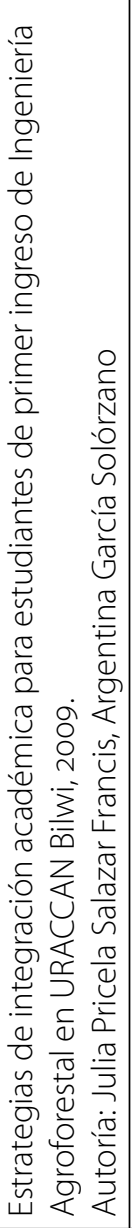 & 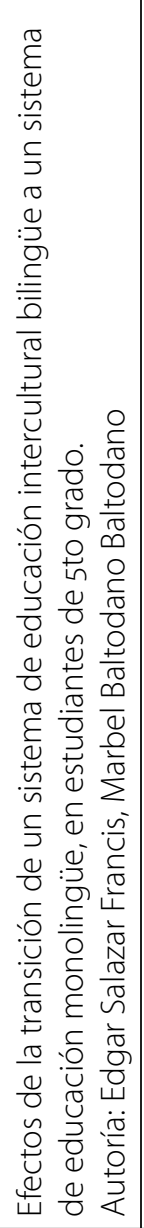 & 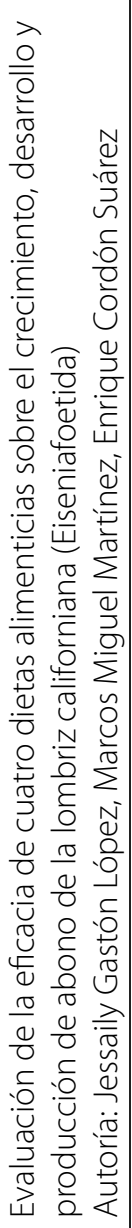 & 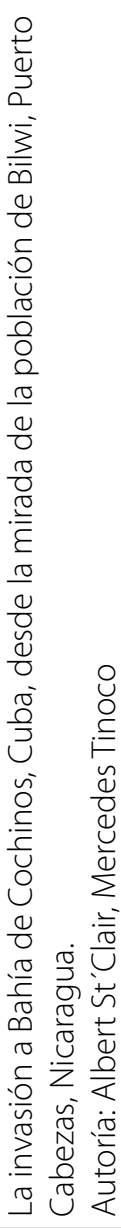 \\
\hline 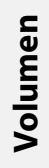 & $\frac{\tilde{F}}{\stackrel{\text { }}{N}}$ & $\frac{\underset{\check{D}}{\stackrel{\Gamma}{m}}}{\frac{m}{m}}$ & $\frac{\stackrel{N}{O}}{\frac{N}{N}}$ & 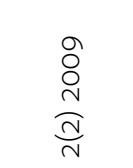 & 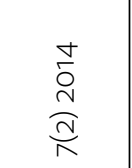 & 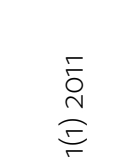 & 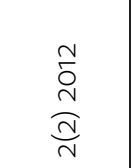 & $\begin{array}{l}\frac{\bar{c}}{D} \\
\underset{N}{N}\end{array}$ \\
\hline 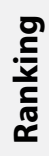 & $\stackrel{m}{q}$ & f & $\checkmark$ & 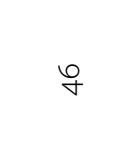 & 于 & q & $\checkmark$ & in \\
\hline
\end{tabular}




\section{Conclusiones}

Es un hecho que las revistas electrónicas superan a las revistas impresas; para ello, los requerimientos y condiciones necesarias para su expansión están a la disposición de las instituciones y organizaciones gestoras de las revistas, por ejemplo, existen programas diseñados de acceso libre para su montaje, siendo el ejemplo el software de código abierto para la gestión de revistas llamado Open Journal Systems (OJS), el que ha sido desarrollado por el proyecto Public Knowledge de la Universidad de British Columbia en Canadá. La demanda por parte de los usuarios es creciente, se dispone de suficientes medios para la difusión de la producción científica y las revistas electrónicas representan una significativa disminución de los costos de publicación.

En la URACCAN son evidentes los resultados obtenidos con la publicación del Portal de Revistas y la tremenda difusión de la revista digital Ciencia e Interculturalidad, entre ellos sobresale:

- Incorporada al Portal de Revistas Nacionales de Nicaragua.

- En catálogo del portal del Sistema regional de información en línea para revistas científicas de América Latina, el Caribe y Portugal (LATINDEX).

- Incorporada en el portal de Latin American Journals Online (LAMJOL).

- Presente en el Portal de Portales LATINDEX que aglutina a 18 portales.

- En Latin American Jornal Online se identificó como una de las tres revistas más consultadas en el período de agosto de 2010 a junio de 2012 y dos de sus artículos se posicionaron entre los 10 más leídos. En el período de marzo del 2011 a octubre del 2013, la Revista Ciencia e Interculturalidad recibió un total de 53,757 revisiones de sus artículos. Las consultas trimestrales más altas se dieron en el primero y segundo trimestre del 2013 con 9,787 y 9,813 respectivamente.

- En el Portal de Revistas de Nicaragua, en el período de 2012 a noviembre del 2013, recibió un total de 12,522 visitas en revisión a resúmenes y 13,881 para revisar o descargar artículo completo en PDF.

- En el portal de Revistas de la URACCAN, en el período de junio del 2011 a octubre del 2013, Ciencia e Interculturalidad tiene un promedio mensual de 1,152 visitas para revisar o descargar artículos completos y 666 para revisar resúmenes. Referente a visitas totales tiene un total de 18,661 visitas en revisión a resúmenes y 32,254 para revisar o descargar artículo completo en PDF.

- El 12 de noviembre del 2013, el Consejo Nicaragüense de Ciencia y Tecnología de Nicaragua (CONICYT), entregó a la Universidad de las Regiones Autónomas de la Costa Caribe Nicaragüense (URACCAN), un reconocimiento a la revista científica Ciencia e Interculturalidad, que textualmente dice: "por su esfuerzo 
en elevar la calidad y aplicación de los criterios del Sistema Regional de Información en Línea, para revistas científicas de América Latina, el Caribe, España y Portugal (LATINDEX) y lograr visibilidad de la producción científica de Nicaragua en Latin American Journal Online (LAMJOL)".

\section{Lista de referencia}

INASP. 2012. El Boletín LAMJOL. http://www.inasp.info/uploads/filer_public/2013/o4/og/lamjol_newsletter_1_spanish.PDF

Sioux Cumming. 2013. Estadísticas Ciencia e Interculturalidad en Latin American Journal Online (LAMJOL). Documento digital. 